\title{
PHYTOBENTHOS ET PRODUCTIVITÉ PRIMAIRE D'UN LAC DE HAUTE MONTAGNE DANS LES PYRENÉES CENTRALES
}

\author{
par J. CapblancQ ${ }^{1}$.
}

\begin{abstract}
L'importance des communautis algales benthiques dans la productivité primaire d'un lac de haute montagne est évaluée. Les algues lithophytiques de la zone littorale $(0-6 \mathrm{~m})$ et Nitella flexilis, qui recouve pratiquement tout le fond de la zone littori-profonde $(6-19 \mathrm{~m})$, forment une biomasse environ 140 fois supérieure à la biomasse movenne de phytoplancton pendant la période estivale. Nitella flexilis constitue $80 \%$ de la biomasse algale benthique; les variations de sa teneur en Chlorophylle traduisent une adaptation aux modifications du climat lumineux qu'elle détermine en partie. La colonisation de substrats artificiels s'avère peu adéquate pour évaluer la production du périphyton; elle indique cependant une productivité 10 fois moindre en zone littorale qu'en zone littori-profonde. Les mesures, au 14C, du taux d'assimilation de Nitella flexilis et des algues épiphytes révèlent qu'à la suite de phénomènes de compensation par le développement du périphyton et lajustemont de la teneur en Chlorophylle, l'activité photosynthétique dépend peu de la lumière. La contribution des algues benthiques à la production piimaire est évaluće à $30 \%$. Leur rô!e dans le cycle des éléments chimiques est envisagé.
\end{abstract}

\section{Phytobenthos and primary production of a high mountain lake in the central Pyrenees.}

The importance of the benthic algal community in the primary productivity of a high moutain lake is evaluated. The lithophytic algae of the littoral zone $(6-19 \mathrm{~m})$, form a biomass about 140 times greater than the mean biomass of the phytoplankton during the summer. Nitella flexilis forms $80 \%$ of the benthic algal biomass; the variations in its chlorophyll content show an adaptation to the light climate which it partly determines. The colonisation of artificial substrata was found to be not very adequate for cvaluating the production of periphyton; it indicated a productivity 10 times less in the littoral zone than in the littoral-profundal zone. Measurements, with $14 \mathrm{C}$, of the rate of assimilation of Nitella fexilis and epiphytic algae revealed that, as a consequence of the phenomena of compensation by the development of periphyton and the adjustment of chlorophyll content, photosynthetic activity is not very dependent on light. The contribution of benthic algae to the primary production is estimated to be $30 \%$. Their role in the cycling of chemical elements is considered.

1. Cette note constitue une partie d'une thèse présentée devant l'Université Paul-Sabatier de Toulouse en vue de l'obtention du Doctorat ès Sciences Naturelles.

Laboratoire d'Hydrobiologie, Université Paul-Sabatier, 118, route de Narbonne, 31077 Toulouse Cedex. 


\section{INTRODUCTION}

Si les producteurs primaires constituent, dans leur ensemble, le niveau trophique le plus intensément étudié dans les écosystèmes aquatiques, la majorité des mesures de productivité primaire concernent la zone pélagique. La distribution relativement homogène des algues planctoniques et l'introduction de techniques de mesures sensibles, telle la méthode au ${ }^{14} \mathrm{C}$, pour évaluer le taux de croissance des communautés phytoplanctoniques ont favorisé sans nul doute la multiplication de ces mesures en limnologie et en océanographie. Inversement, l'hétérogénéité des communautés végétales benthiques, leur distribution irrégulière sur les substrats naturels et leur dépendance vis-à-vis de ces substrats constituent un obstacle essentiel au développement de méthodes de mesures quantitatives comparables à celles qui existent pour le phytoplancton. La contribution de la flore benthique des régions littorales et profondes à la productivité primaire totale des écosystèmes aquatiques est par suite souvent négligée. Quelques exemples (Wetzel 1964 a; Pieczynska et Szczepanska 1966; Allen 1971; Kajak et al. 1972) montrent cependant que la productivité des communautés végétales sessiles, généralement sous-estimée, excède celle du phytoplancton dans certains lacs peu profonds.

Les études semi-quantitatives ou quantitatives sur les macrophyles (rerues par Gessner 1955 et 1959) et les algues sessiles (revues par Cooke 1956, Sladeckova 1962 et Wetzel 1964 a) sont très nombreuses. Plusieurs travaux concernent des déterminations de biomasse, son évolution dans le temps ayant donné lieu à quelques interprétations en termes de productivité (Sladecek et Sladeckova 1964; Kevern et al. 1966; Castenholz 1960 pour le périphyton; Westlake 1963 et 1965 pour les macrophytes). Selon Wetzel 1965) il est à peu près certain que les taux de production donnés par cctte méthode sont largement inférieurs à ceux obtenus par les mesures «in situ» de productivité primaire. Ces dernières restent cependant relativement rares. L'utilisation des échanges gazeux $\left(\mathrm{O}_{2}, \mathrm{CO}_{2}\right)$ en enceintes closes est limitée par des problèmes tels que le stockage des gaz dans le système lacunaire des macrophytes (Hartman et Brown 1967) ou l'impossibilité de dissocier l'activité respiratoire des sédiments du métabolisme des végétaux. Quelques auteurs ont utilisé la technique $d u{ }^{14} \mathrm{C}$ pour mesurer l'activité photosynthétique de communautés algales dévelorpées sur des substrats artificiels (Vollenweider et Samaan 1958; Backhaus 1967; Allen 1971), des algues benthiques des plages sabloneuses (Baird et Wetzel 1968; Grøntved 1960; Hunding 1971; Hunding et Hardgrave 1973) ou de fragments de macrophytes (Bodin 
et Nauwerck 1968). WVetzel (1964 a) a mesuré l'assimilation du ${ }^{14} \mathrm{C}$ dans des cloches en plexiglas posées sur le fond; cette méthode lui permet d'étudier l'activité photosynthélique des macrophytes et des algues épilithiques dans des conditions aussi proches que possible des conditions naturelles. Ellc n'est malheureusement applicable que dans des caux peu profondes où la nature du fond se prête à la mise en place de ces cloches. Dans la plupart des cas, il reste cependant difficile d'extrapoler les résultats de ces mesures à des surfaces de substrat naturel plus importantes (p. ex. au $\mathrm{m}^{2}$ ) par suite de la distribution hétérogène des algues benthiques.

La transparence élevée des eaux lacustres de haute montagne permet le développement des algues benthiques jusqu'à une profondeur élevée. Deux travaux préliminaires (Besch el al. 1972; Backhaus, en prép.) ont montré qu'une flore algale riche et diversifiée colonisait la totalité du lac de Porl-Bielh, les formes dominantes se succédant, en profondeur, en fonction des substrats.

Afin de préciser l'importance de ces communautés algales benthiques dans la productivité primaire d'un lac de haute montagne, il nous a paru intéressant de compléter l'étude du phytoplancton (Capblancq 1972) par des mesures quantitatives de la biomasse el de la productivité des algues du fond au lac de Port-Bielh $(2288 \mathrm{~m}$ ).

\section{MATÉRIEL ET MÉTHODES}

\subsection{Biomasse.}

La biomasse a été déterminée sur des échantillons prélevés après le dégel (début juillet) et en automne (fin septembre). Dans la zone littorale du lac, les algues épilithiques ont élé recueillies par bressage de la surface de cailloux de granite de 10 à $15 \mathrm{~cm}$ prélevés entre $0 \mathrm{~m}$ el $6 \mathrm{~m}$ de profondeur. La surface d'échantillonnage (100 à $400 \mathrm{~cm}^{2}$ ) a été déterminéc ensuite par contact avec un papier calque. Dans la zone littori-profonde, 98 prélèvements de Nitella ont été réalisés au moyen d'une drague Ekmann. Le fonctionnement de cet appareil dans les touffes de Nitella a pu être apprécié depuis la surface jusqu'à une profondeur de 10 à $11 \mathrm{~m}$ par suite de la transparence élevée des eaux du lac. Les végélaux correspondant à une surface de prélèvement de $1 / 45 \mathrm{~m}^{2}$ ont été lavés sur place à travers un filet fin. Les échantillons de Nitella ont été pesés après dessication à l'étuve à $105{ }^{\circ} \mathrm{C}$ pendant 24 heures (poids sec) et après ignition au four à $550^{1 \circ} \mathrm{C}$ pendant une heure (poids de cendres). Les algues épilithiques ont été concentrées par centrifugation à 5500 tours/minute pendant 15 minutes. Le culot de 
centrifugation a été partagé en deux sous-échantillons de poids identique. Le poids sec et le poids de cendres ont été déterminés sur le promier, la teneur en chlorophylle sur le second.

\subsection{Pigments chlorophylliens.}

Les dosages de la chlorophylle a du périphyton littoral peuvent être affectés par les produits de dégradation des pigments chlorophylliens des algues mortes ou sénescentes (Wetzel 1964 a). Les pigments du culot de centrifugation des algues épilithiques ont été extraits par broyage aux ultra-sons dans l'acétone à $90 \%$. La densité optique de l'extrait a été détcrminée aux longueurs d'onde de $750 \mathrm{~m} \mu$ et $665 \mathrm{~m} \mu$ (spectrophotomètre Jouan) avant et après acidification. L'équation de Lorenzen (1967) a été utilisée pour le calcul de la concentration en chlorophylle a. Les pigments chlorophylliens des Nitella ont été dosés à 4 dates de l'été 1972 sur des échantillons d'algues prélevés aux profondeurs de $6,8,10,12,14$ et 16 mètres. Pour chaque dosage, trois portions d'échantillon pesées après 10 seconde d'essorage sur papier filtre ont été broyées dans de l'acétone basique à $90 \%$ Le rapport poids frais/poids sec a été déterminé sur le reste de l'échantillon pesé après essorage sur papier filtre et après dessication à l'étuve à $105^{\circ} \mathrm{C}$. La densité optique des extraits a été mesuréc, après centrifugation, aux longueurs d'onde de $750,665,645$ et $630 \mathrm{~m} \mu$. Les calculs des concentrations en chlorophylle $a, b$ et c exprimées par gramme de poids végétal sec, sont basés sur les formules de Strickland el Parsons (1968).

\subsection{Productivité.}

La nature du substrat rocheux en zone littorale et la profondeur élevée de la zone colonisée par les Nitella ne permettaient pas d'envisager la réalisation de mesures « in situ » de l'activité photosynthétique des algues benthiques. Par ailleurs, l'assimilation de ${ }^{14} \mathrm{C}$ par des communautés algales développées sur des substrats artificiels représente une valeur difficilement exploi lable. La durée d'immersion des substrats, nécessaire pour atteindre les conditions climaciques est en effet 1 rès variable selon les caractères du milieu et les espèces concernées. Dans le lac de Port-Bielh, le peuplement des substrats naturels différait qualitativement et quantitativement de celui des substrats artificiels immergés pendant deux mois en zone littorale (Besch et al. 1972). En outre, la conversion des résultals par unité de surface de fond se heurte d'une part au problème de l'estimation des biomasses sur les supports naturels colonisés par les algues (chaos granitique en zone littorale, tiges 
de Nitella en zone profonde) et, d'autre part, aux difficultés liées à la distribution irrégulière des algues sur ces substrats.

L'activité photosynthétique des Nitella et des algues périphy'iques a été mesurée globalement à l'aide de ${ }^{14} \mathrm{C}$ par une méthode analogue à celle utilisée pour le phytoplancton (Capblancq 1972). Des touffes de Nitella, prélevées à différentes profondeurs au moyen d'une drague Petersen, ont été placées dans des flacons transparents et opaques de $150 \mathrm{ml}$ remplis d'eau prélevéc au contact $\mathrm{du}$ fond. Après addition d'un $\mathrm{ml}$ de ${ }^{14} \mathrm{CO}_{3} \mathrm{NaH}$ d'une activité de $1 \mu \mathrm{Ci}$, les flacons ont été immergés aux profondeurs de prélèvement pour une durée de 4 à 6 heures symétrique par rapport au midi solaire. Les tiges de Nitella récupérées à la fin de l'expérience ont été immédiatement essorées sur papier filtre et séchées au dessicateur à l'obscurité. Des portions de poids connu (2 à $6 \mathrm{mg}$ ) de ces échantillons, réduits au préalable en poudre fine par broyage au mortier, ont été fixées sur l'envers d'étiquettes autocollantes pour les mesures de radioactivité. L'autoabsorption des radiations par les tissus végétaux a été déterminée en comptant, de la même façon, des quantités de poudre de poids croissant. L'extrapolation de la courbe obtenue (fig. 1) donne l'activité de l'échantillon pour une épaisseur nulle de matériel végétal.

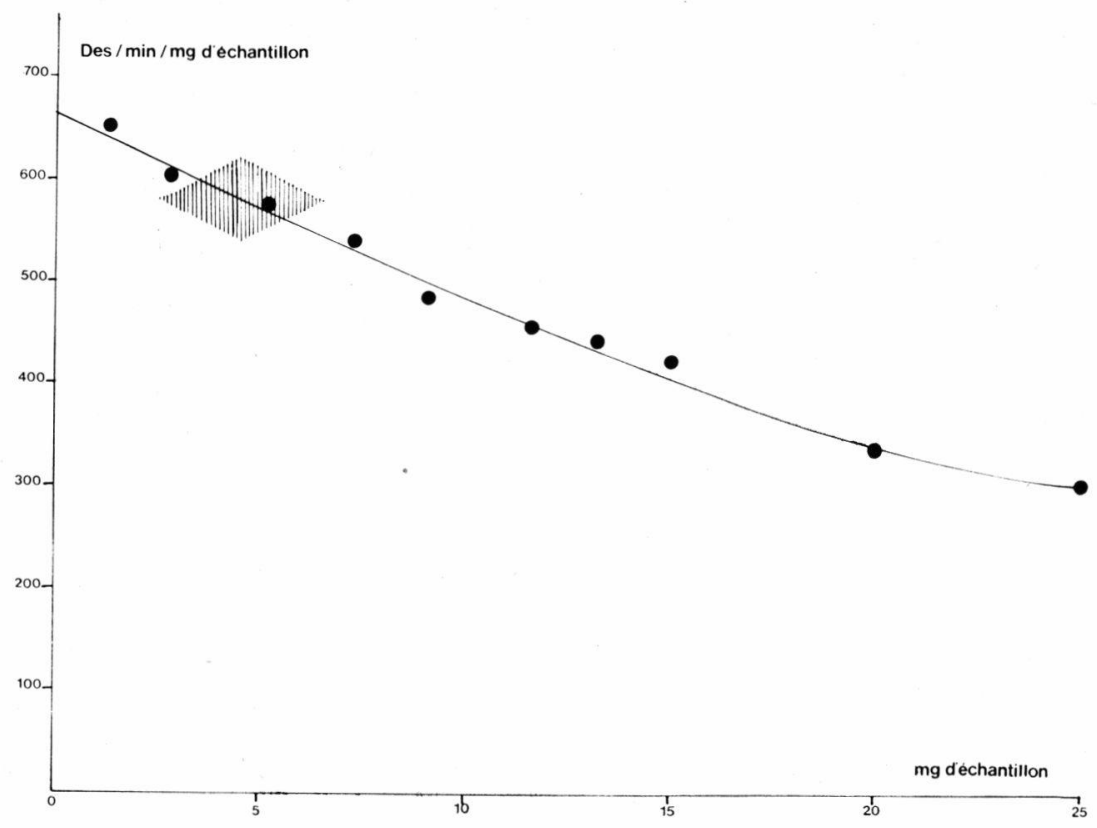

FIG. 1. - Courbe d'autoabsorption de Nitella flexilis. La zone hachurée indique les conditions de comptage des échantillons lors des mesures de productivité. 
Nous avons tenté d'évacuer la productivité des algues périphytiques et épilithiques en déterminant le taux de colonisation de substrats artificiels immergés à différentes profondeurs du lac. Cette méthode, utilisée par plusieurs auteurs (Castenholz 1960 et 1961; Sladecek et Sladeckova 1964; Kevern et al. 1966; Maciolek et Kennedy 1964; Neal et al. 1967; Dumont 1969; Stockner et Armstrong 1971; Welch et al. 1972), mesure la biomasse accumulée par fixation et croissance des algues moins les pertes par respiration, mort et décrochage. Si les valeurs obtenues peuvent être entachées par de nombreuses sources d'erreurs (sélectivité des substrats artificiels, perte de matériel lors des manipulations, influence de la position des substrats et de la durée d'exposition sur la vitesse de colonisation) elles fournissent toutefois une indication sur la productivité relative des communautés périphytiques aux différentes profondeurs. Six substrats artificiels ont été immergés le 9 juillet 1972. Chaque substrat se composait de 2 cylindres en plexiglas disposés en croix et lestés, sur lesquels étaient fixées 1. feuilles de polyéthylène de $25 \times 25 \mathrm{~cm}$ (fig. 2). Ces substrats, placés au contact du fond à $1,4,6,9,13$ et 16 mètres de profondeur, ont été relevés tous les mois pour prélever une feuille de polyéthylène. Cinq carrés de $1 \mathrm{~cm}$ de côté ont été découpés sur chaque feuille et montés directement entre lame et lamelle pour un comptage direct, au microscope, des algues fixées. Le volume moyen des cellules ou des colonies des diverses espèces a été

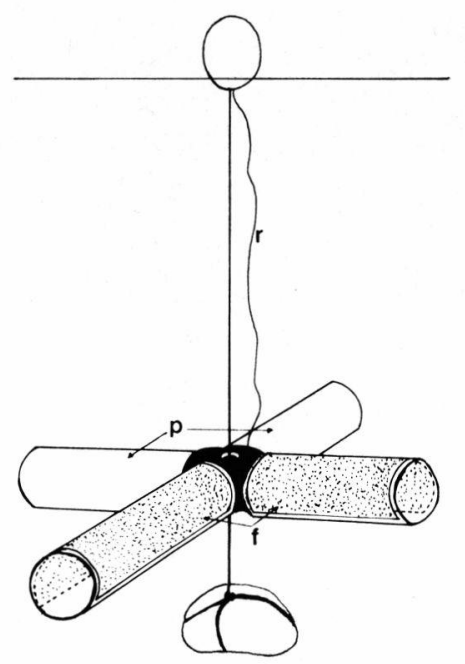

FIG. 2. - Schéma des supports en plexiglas utilisés pour les substrats artificiels. f : feuille de polyéthylène; $p$ : cylindres de plexiglas; $r$ : corde de rappel. 


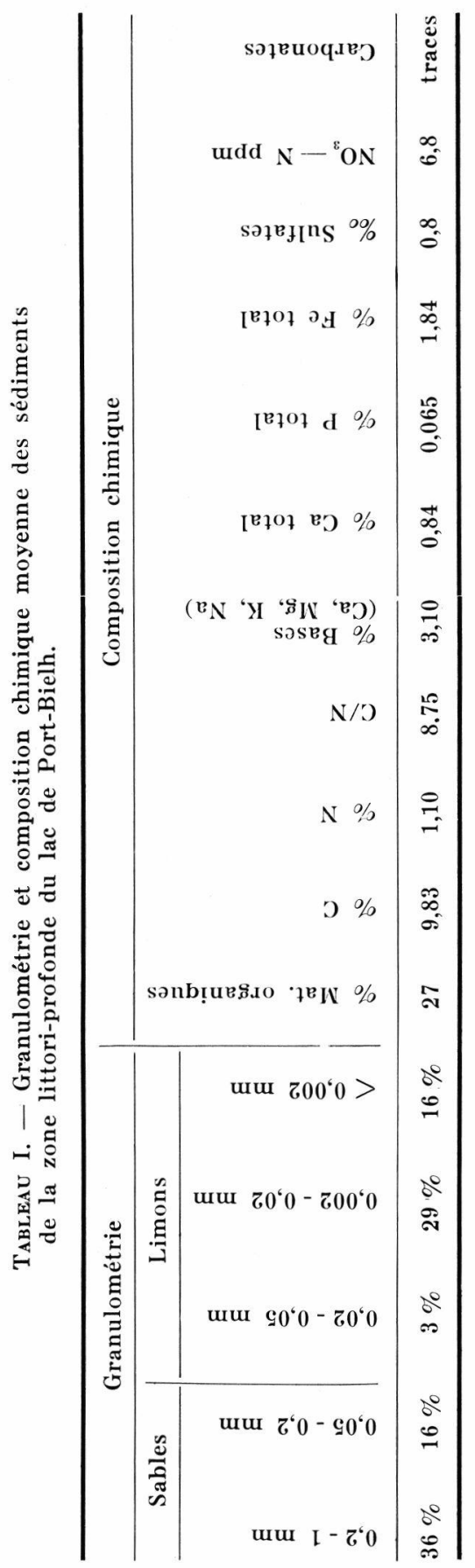


calculé el le nombre moyen de cellules $/ \mathrm{cm}^{2}$ converti en biovolume. La chlorophylle du bioderme, prélevé par brossage du reste de la feuille, a été dosée par la méthode trichromatique utilisée pour les Nitella. Las algues développées sur les cylindres de plexiglas libérés des feuilles de polyéthyline ont été recueillies à la fin de la période d'exposition el concentrées par centrifugation à 6000 tours $/ \mathrm{mn}$ pendant 20 minu!es. Le volume (mensuration du culot de centrifugation), le poids sec et le poids de cendres des culots de centrifugation ont été déterminés afin d'établir les relations entre ces trois paramètres.

\section{RESULTATS}

\subsection{Caractéristiques du fond du lac de Port-Bielh.}

Les caractéristiques morphométriques et physico-chimiques du lac de Port-Bielh ont été définies dans les travaux précédents (Capblancq et Laville 1968 et 1972; Capblancq 1972). Le fond de ce lac transparent se caractérise par la succession en profondeur de deux zones de structure différente :

a) La zone littorale ceinture le lac jusqu'à une profondeur de 6 à $7 \mathrm{~m}$ et, avec une superficie de 6 ha, représente $36 \%$ de la surface totale du fond. Le substrat rocheux est constitué d'un enchevêtrement de gros blocs d'éboulis granitiques recouverts d'une mince pellicule de vase fine et d'algues épilithiques. Une vase riche en débris végétaux d'origine exogine s'accumule de place en place entre les blocs rocheux. Dans une anse de la partie sud du lac, une population d'Isoetes brochoni Motelay occupe une surface restreinte de quelques mètres carrés. Les communautés algales visibles à l'oil nu (Besch et al. 1972) permettent de reconnaître :

- une zone de Schizothrix-Calothrix jusqu'à $0,50 \mathrm{~m}$ de profondeur (zone «eulittorale» sensu Ruttner 1962),

- une zone de Nostoc comprise entre $0,50 \mathrm{~m}$ et selon les points, 1 à $5 \mathrm{~m}$ de profondeur (zone «infralittorale» sensu Ruttner).

b) La zone littori-profonde (Capblancq el Laville 1972) se caractérise par une accumulation de vase finc, riche en matières organiques. Sa composition granulométrique el ses caractères chimiques sont récapitulés dans le tableau $I$. Celle zone est entièrement colonisée par une prairie de Nitella flexilis Linné dont la densité atteint un maximum entre 12 et $15 \mathrm{~m}$ de profondeur. Durant les 6 mois de stagnation hivernale l'eau est pratiquement dépourvue d'oxygène dissous au contact du fond. 
(9)

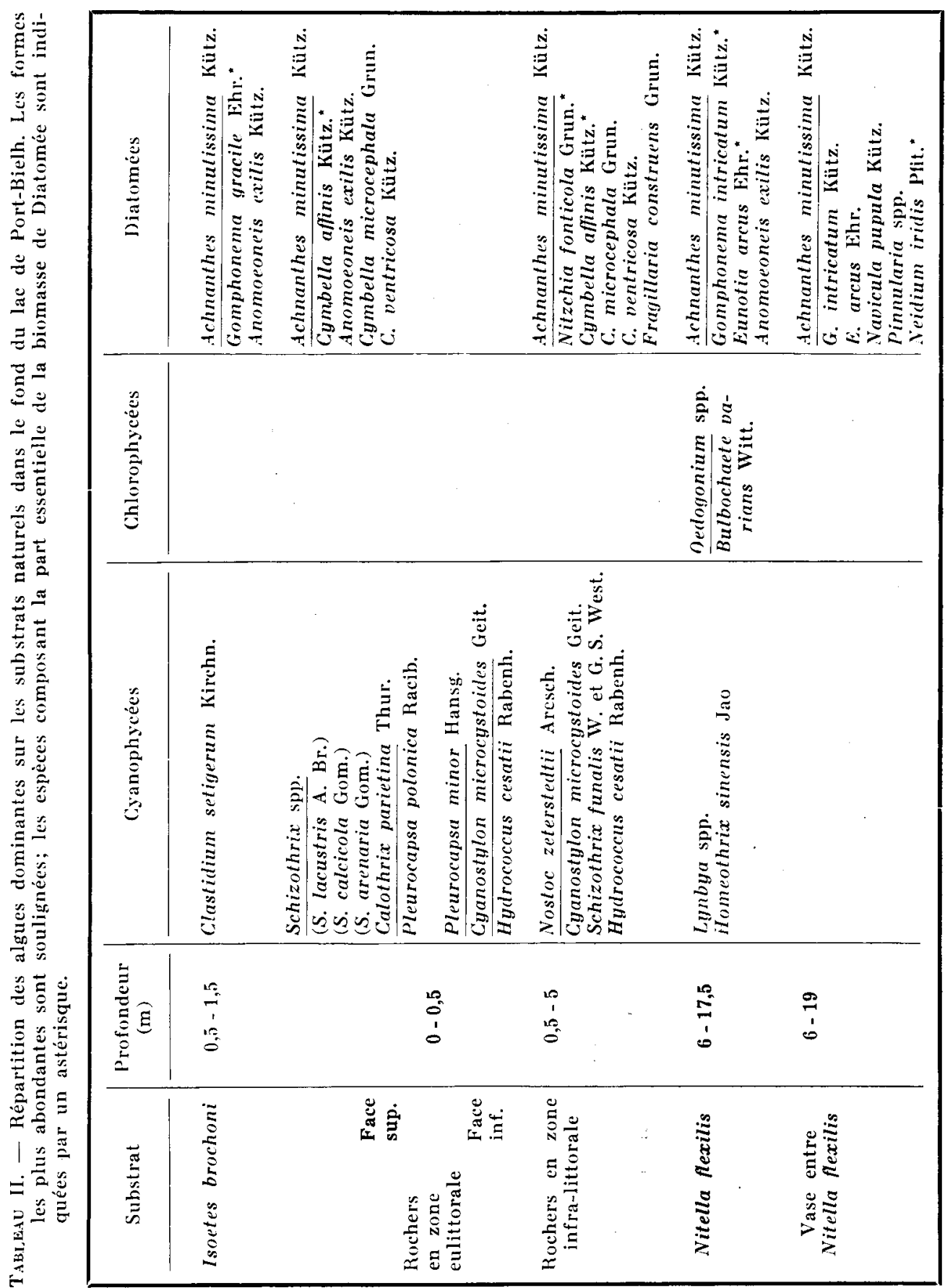




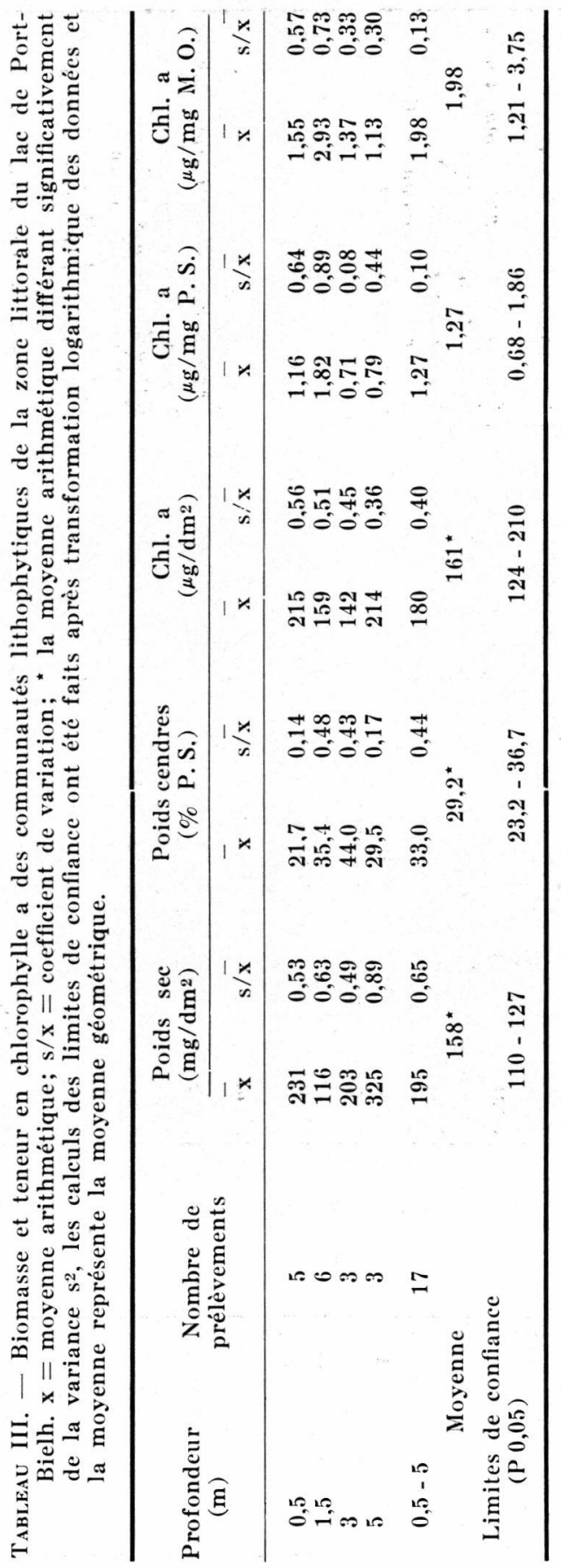




\subsection{Les algues benthiques : distribution et biomasse.}

Les listes floristiques et la répartition des algues benthiques du lac de Port-Bielh sont données dans les travaux de Besch et al. (1972) et Backhaus (en prép.). Nous nous bornerons à rappeler ici les caractères essentiels de la distribution des espèces dominantes dans le fond du lac (tableau II).

Le peuplement se caractérise par une diversité spécifique relativement élevée (140 espèces) si on le compare à celui de la zone pélagique (Capblancq 1972). Les algues bleues (Nostoc zetterstedtii, Lyngbya sp., Schizothrix spp., Calothrix spp.) et vertes (Nitella flexilis, Oedogonium sp., Bulbochaete varians) constituent une part importante de la biomasse algale alors qu'elles jouent un rôle négligeable dans les communautés phytoplanctoniques. Quelques espèces (Achnanthes minutissima, Gomphonema intricatum), numériquement abondantes, colonisent tous les substrats à toutes les profondeurs. Généralement, les espèces benthiques se Iocalisent sur un type de substrat et se répartissent en zones nettes aux différentes profondeurs du lac (tableau II).

Les prélèvements réalisés sur les rochers de la zone littorale $(0,50 \mathrm{~m}-5 \mathrm{~m})$ le 9 -VII-72 (9 prélèvements) et le 10-X-72 (8 prélèvements) permettent d'estimer entre 11 et $22 \mathrm{~g}$ de poids $\mathrm{sec} / \mathrm{m}^{2}$ de substrat la biomasse moyenne de matériel fixé soit environ 8 à $15 \mathrm{~g}$ de matières organiques $/ \mathrm{m}^{2}$. La quantité moyenne de chlorophylle a est de l'ordre de $16,1 \mathrm{mg} / \mathrm{m}^{2}$ de substrat. Il n'existe aucune différence significative entre les valcurs mesurées aux différentes profondeurs (tableau III) ainsi qu'entre les prélèvements réalisés au début et à la fin de l'été. Les variations de poids entre les divers prélèvements sont relativement importantes et dépendent de la quantité de détritus (vase) et de colonies de Nostoc présente sur les cailloux prélevés. Les relations entre poids sec, poids de cendres et quantité de chlorophylle a sont également très lâches. Comparées aux valeurs moyennes admises par Sladecek et Sladeckova (1964) d'après les données de plusieurs auteurs, le pourcentage moyen de cendres ( 23 à $37 \%$ ) est relativement élevé, et inversement, le pourcentage de chlorophylle a $(0,13 \%)$ est nettement plus faible. Ces différences sont attribuables à l'importance de matériel minéral et organique non chlorophyllien présent sur la surface des pierres immergées. La vase, plus ou moins riche en matières organiques, peut abriter des quantités variables d'organismes hétérotrophes (bactéries, champignons, protozoaires, larvules d'insectes ...). Par ailleurs, la présence des colonies de Nostoc, qui se développent en agrégats sur la face supérieure de certaines pierres, se traduit par une diminution importante du pourcentage 
de cendres et de chlorophylle a. La dispersion des mesures traduit une assez grande hétérogénéité dans la composition et la distribution des communautés litophytiques.

Nitella flexilis Linné apparaît à partir de -6 à -7 mètres lorsque le substrat rocheux fait place à une couche de vase fine. La longueur et la densité des tiges augmente progressivement avec la profondeur jusqu'à une zone comprise entre -12 et $-15 \mathrm{~m}$ où la biomasse de cette algue peut être estimbée à $61,5 \stackrel{\times}{\stackrel{\leftarrow}{\div}} 1,27 \mathrm{~g}$ de poids $\mathrm{sec} / \mathrm{m}^{2}$ de fond. Les résultats de 98 prélèvements réalisés entre 6 et $18 \mathrm{~m}$ sont résumés dans le tableau IV et schématisés dans la figure 3 . La distribution quantitative de Nitella flexilis en fonction de la profondeur rappelle celle précédemment décrite pour le phytoplancton dont les biomasses maximales se situent entre 12 et $16 \mathrm{~m}$ (Capblancq 1972). D'autre part, ces prélèvements permettent d'estimer à $20,3 \pm 1,8 \%$ le rapport poids de cendres/poids sec.

TABLeau IV. - Nitella flexilis : biomasse aux diverses profondeurs du lac de Port-Bielh; teneur en cendres et rapport Poids sec/Poids frais : moyenne arithmétique des prélèvements et limites de confiance au seuil $5 \%$ (" les limites inférieure et supérieure de la moyenne ont été calculées après transformation logarithmique des données).

\begin{tabular}{|c|c|c|c|c|}
\hline $\begin{array}{l}\text { Profondeur } \\
\quad(\mathrm{m})\end{array}$ & $\begin{array}{l}\text { Nombre de } \\
\text { prélèvements }\end{array}$ & $\begin{array}{l}\text { Superficie } \\
(\text { ha })\end{array}$ & $\begin{array}{l}\text { Poids sec } \\
\left(\mathrm{g} / \mathrm{m}^{2}\right)\end{array}$ & $\begin{array}{l}\text { Poids total } \\
\quad(\mathrm{kg})\end{array}$ \\
\hline $6-9$ & 23 & 3,25 & $\begin{array}{c}15,98 \\
(7,72-33,08)^{\star}\end{array}$ & $\begin{array}{c}519 \\
(251-1075)\end{array}$ \\
\hline $9-12$ & 24 & 3,37 & $\begin{array}{c}42,55 \\
(29,75-60,85)^{\star}\end{array}$ & $\begin{array}{c}1434 \\
(1002-2050)\end{array}$ \\
\hline $12-15$ & 28 & 2,71 & $\begin{array}{c}61,63 \\
(48,53-78,27)^{*}\end{array}$ & $\begin{array}{c}1670 \\
(1315-2121)\end{array}$ \\
\hline $15-17$ & 17 & 1,19 & $\begin{array}{c}41,55 \\
(27,33-63,15)^{*}\end{array}$ & $\begin{array}{c}494 \\
(325-751)\end{array}$ \\
\hline $17-19$ & 6 & 0,98 & $\left(-^{0}-\right)$ & $\left(-^{0}-\right)$ \\
\hline $\mathbf{\Sigma}$ & 98 & 11,50 & & $\begin{array}{c}4117 \\
(2893-5997)\end{array}$ \\
\hline \multirow{2}{*}{\multicolumn{3}{|c|}{$\begin{array}{l}\text { Cendres }(\% \text { Poids sec })= \\
\text { Poids sec }(\% \text { Poids frais })=\end{array}$}} & $20,3 \%$ & $(18,5 \%-22,1 \%)$ \\
\hline & & & $8,64 \%$ & $(8,22 \%-9,04 \%)$ \\
\hline
\end{tabular}

Les pigments chlorophylliens des Nitella et des algues périphytiques sont indissociables. Les résultats des dosages effectués à 4 dates de l'été 1972 sont rassemblés dans le tableau V. Ils révèlent : 
Taridau V. - Nitela flexilis : teneur en chlorophylle a et b (mg Chl./g P.S.) et rapport $a / b$ à diverses profondeurs et à quatre dates différentes. Chaque valeur représente la moyenne de trois dosages.

\begin{tabular}{|c|c|c|c|c|c|c|c|c|}
\hline Date & Chl. & $6 \mathrm{~m}$ & $8 \mathrm{~m}$ & $10 \mathrm{~m}$ & $12 \mathrm{~m}$ & $14 \mathrm{~m}$ & $16 \mathrm{~m}$ & Moyennes \\
\hline 4-VIII-72 & $\begin{array}{c}a \\
b \\
a / b\end{array}$ & $\begin{array}{l}1,47 \\
1,36 \\
1,08\end{array}$ & $\begin{array}{l}1,43 \\
0,85 \\
1,68\end{array}$ & $\begin{array}{l}1,41 \\
0,86 \\
1,64\end{array}$ & $\begin{array}{l}1,35 \\
1,18 \\
1,14\end{array}$ & $\begin{array}{l}3,20 \\
2,21 \\
1,45\end{array}$ & $\begin{array}{l}1,90 \\
1,76 \\
1,08\end{array}$ & $\begin{array}{l}1,79 \\
1,37 \\
1,32\end{array}$ \\
\hline 23-VIII-72 & $\begin{array}{c}a \\
b \\
a / b\end{array}$ & $\begin{array}{l}1,48 \\
0,85 \\
1,80\end{array}$ & $\begin{array}{l}1,51 \\
1,63 \\
0,92\end{array}$ & $\begin{array}{l}2,07 \\
1,63 \\
1,27\end{array}$ & $\begin{array}{l}2,69 \\
1,82 \\
1,48\end{array}$ & $\begin{array}{l}3,01 \\
1,14 \\
2,64\end{array}$ & $\begin{array}{l}\mathbf{3}, 50 \\
1,62 \\
2,16\end{array}$ & $\begin{array}{l}2,37 \\
1,45 \\
1,71\end{array}$ \\
\hline $18-\mathrm{IX}-72$ & $\begin{array}{c}a \\
b \\
a / b\end{array}$ & $\begin{array}{l}3,12 \\
1,04 \\
3,00\end{array}$ & $\begin{array}{l}2,78 \\
0,97 \\
2,86\end{array}$ & $\begin{array}{l}2,49 \\
0,95 \\
2,62\end{array}$ & $\begin{array}{l}2,76 \\
1,01 \\
2,73\end{array}$ & $\begin{array}{l}3,08 \\
1,13 \\
2,72\end{array}$ & $\begin{array}{l}3,79 \\
1,63 \\
2,32\end{array}$ & $\begin{array}{l}3,00 \\
1,12 \\
2,71\end{array}$ \\
\hline $10-X-72$ & $\begin{array}{c}\mathbf{a} \\
\mathbf{b} \\
\mathbf{a} / \mathbf{b}\end{array}$ & $\begin{array}{l}4,02 \\
1,45 \\
2,77\end{array}$ & $\begin{array}{l}3,51 \\
1,37 \\
2,56\end{array}$ & $\begin{array}{l}3,78 \\
1,49 \\
2,53\end{array}$ & $\begin{array}{l}3,09 \\
1,24 \\
2,49\end{array}$ & $\begin{array}{l}4,13 \\
1,54 \\
2,68\end{array}$ & $\begin{array}{l}3,93 \\
1,44 \\
2,73\end{array}$ & $\begin{array}{l}3,74 \\
1,42 \\
2,63\end{array}$ \\
\hline Moyennes & $\begin{array}{c}\mathbf{a} \\
\mathbf{b} \\
\mathbf{a} / \mathbf{b}\end{array}$ & $\begin{array}{l}2,52 \\
1,17 \\
2,16\end{array}$ & $\begin{array}{l}2,31 \\
1,20 \\
2,00\end{array}$ & $\begin{array}{l}2,44 \\
1,23 \\
2,01\end{array}$ & $\begin{array}{r}2,47 \\
1,31 \\
1,96\end{array}$ & $\begin{array}{l}3,35 \\
1,25 \\
2,37\end{array}$ & $\begin{array}{l}3,28 \\
1,61 \\
2,07\end{array}$ & \\
\hline
\end{tabular}

- une augmentation de la quantité de chlorophylle a par unité de poids végélal sec au-delà de $13 \mathrm{~m}$, zone de développement optimal de cette algue.

- une augmentation progressive de la quantité de chlorophylle a par unité de poids végétal sec entre le début du mois d'août et le mois d'octobre.

- une diminution corrélative du rapport chlorophylle a/chlorophylle $b$ au cours de l'été. La valeur de ce rapport double entre le début du mois d'août (1,32 en moyenne) et le mois d'octobre (2,63 en moyenne) à toutes les profondeurs.

On peut penser que ces variations constituent un phénomène d'adaptation des Nitella aux variations des conditions lumineuses. Gessner (1955) indique qu'il existe une relation inverse entre la quantité de chlorophylle et la lumière chez les algues marines en profondeur ou dans des cultures de Chlorelles. Selon cet auteur, le quotient chlorophylle a/chlorophylle b est généralement plus faible chez les algues vertes benthiques marines (1,4 en moyenne) que chez les plantes submergées ( 2,3 en moyenne) ou les plantes terrestres $(4,4$ en moyenne). Il attribue ce phénomène à l'augmentation relative de la quantité de chlorophylle $b$ qui favorise une meilleure absorption de la lumière verte de profondeur. Il n'est cependant pas exclu, dans le cas du lac de Port-Bielh, que les modifications du rapport $a / b$ reflètent les changements qualitatifs et quantitatifs des communautés de la microflore algale associée aux Nitella. Les résultats des dosages des pigments chlorophylliens des 
algues fixées sur les substrats artificiels montrent que la valeur du quotient $\mathbf{a} / \mathbf{b}$ varie en même temps que la structure des communautés algales.

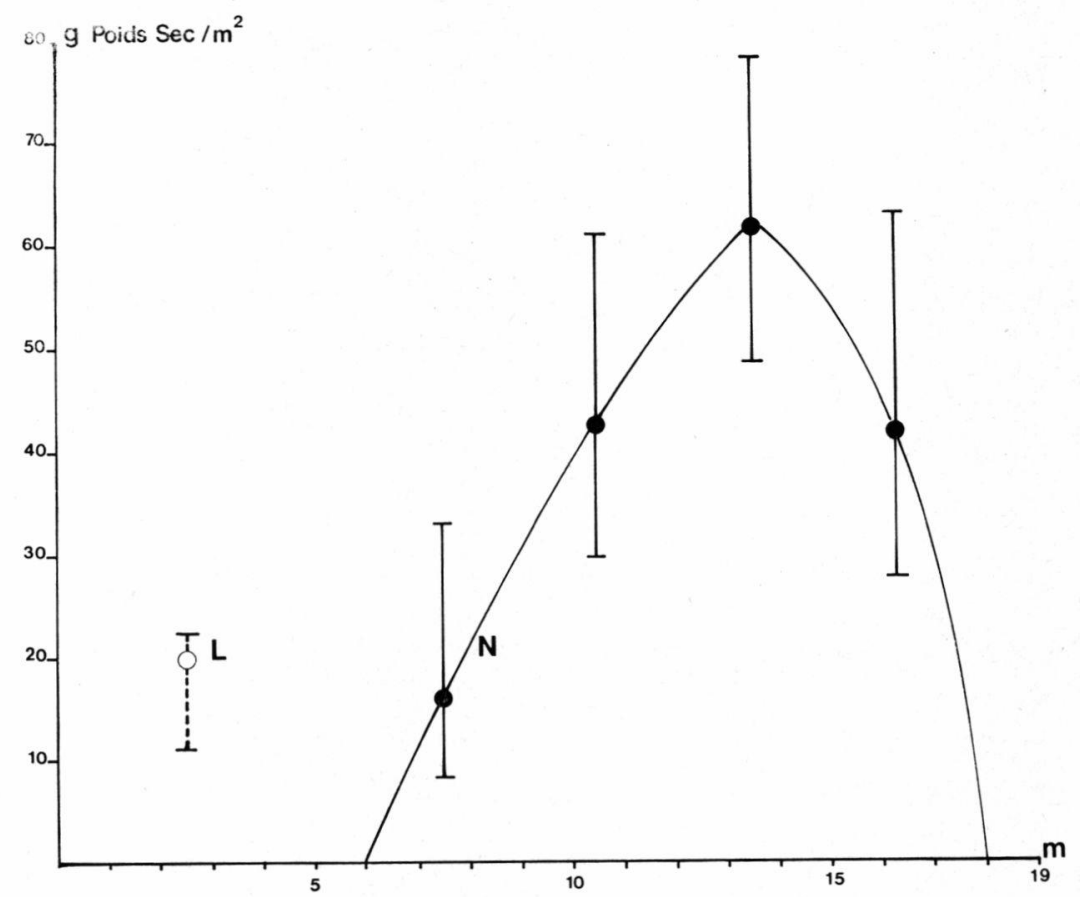

Fig. 3. - Distribution de la biomasse d'algues benthiques sur le fond du lac de Port-Bielh (moyennes arithmétiques et limites de confiance au seuil $5 \%$ ). $\mathrm{L}$ : algues litophytiques; $\mathrm{N}$ : Nitella flexilis.

Les résultats des mesures gravimétriques et des dosages de pigments, pondérés par les surfaces relatives des substrats colonisés, permettent d'estimer entre 24 et $49 \mathrm{~g}$ de poids sec la biomasse moyenne des algues benthiques $/ \mathrm{m}^{2}$ au lac de Port-Bielh. Cette valeur, qui ne tient pas compte des algues épipéliques, s'avère 100 à 200 fois supérieure aux biomasses moyennes de phytoplancton qui se développent dans ce lac en été (Capblancq 1972).

\subsection{Développement des algues benthiques sur les substrats arti- ficiels.}

Les comptages d'algues et les dosages de pigments chlorophylliens, réalisés tous les mois, ont permis de suivre l'évolution des communautés de périphyton établies sur des substrats artificiels immergés juste après le dégel à différentes profondeurs. Les résultats de ces mesures sont résumés dans le tableau VI et la figure 4. 


\subsubsection{CoMPoSITION DU PÉRIPHYTON DES SUBSTRATS ARTIFICIELS.}

La structure des communautés établies sur les feuilles de polyéthylène évolue dans le temps et varie selon la profondeur d'immersion. Les Diatomées sont les premières à coloniser les supports artificiels. Après un mois, elles forment la quasi totalité de la biomasse algale, exception faite pour les substrats de -9 et - 13 mètres très rapidement envahis par un peuplement de Lyngbya sp. qui disparaît au cours du mois d'août. La densité des Diatomées augmente progressivement avec la durée d'exposition. Après deux mois d'immersion, les chlorophycées se développent sur tous les substrats excepté sur ceux de $\longrightarrow \mathbf{1} \mathbf{~ m}$. La biomasse de ce groupe s'accroît au cours du mois de septembre et atteint, à toutes les profondeurs, une valeur optimale au début du mois d'octobre.

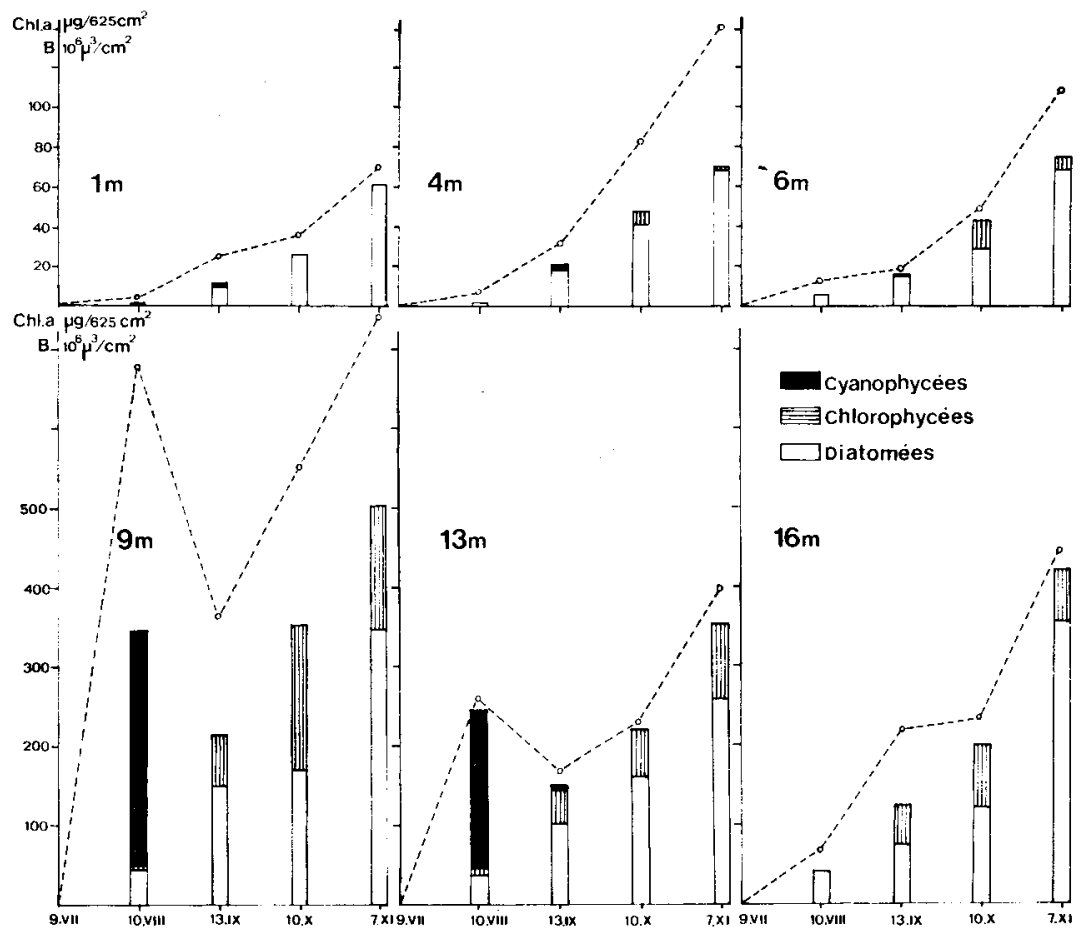

Fig. 4. - Evolution du biovolume de périphyton (histogrammes) et de la quantité de chlorophylle a (courbes en pointillé) sur les substrats artificiels immergés de -1 à $-16 \mathrm{~m}$ dans le lac de Port-Bielh. 
Les Diatomées dominent sur les supports de la zone littorale où elles forment généralement plus de $90 \% \mathrm{du}$ biovolume total. Achnanthes minutissima est l'espèce la plus abondante; elle représente toujours plus de $70 \%$ du biovolume sur les substrats immergés à -1 et -4 mètres. Son abondance relative décroît au-delà de -6 mètres, zone de transition entre les rochers littoraux et la prairie de Nitella. Eunotia arcus et Gomphonema intricatum sont les espèces caractéristiques de la zone littori-profonde. La densité d'E. arcus croît avec la profondeur et devient maximale sur les substrats de $-\mathbf{1 6} \mathrm{m}$. G. intricatum abonde surtout entre -9 et $-13 \mathrm{~m}$; à $-9 \mathrm{~m}$ elle est associée à $G$. constrictum (5 à $15 \%$ du biovolume). Cette dernière, faiblement représentée aux autres profondeurs paraît se substituer ici à $E$. arcus.

Les Chlorophycées, peu développées en zone littorale sont pratiquement limitées aux substrats de la zone littori-profonde où, dès le mois de septembre, elles constituent généralement plus de $25 \%$ du biovolume total. Bulbochaete varians et Oedogonium spp. sont les formes dominantes. La biomasse maximale de $B$. varians apparaît entre le $13-I X$ et le $10-X$ selon les profondeurs. Le développement de Oedogonium est un peu plus tardif; ses plus fortes densités sont atteintes en fin de période d'exposition (7-XI). Coleochaete scutata et Chaetosphaeridium pringsheimii ont une croissance parallèle à celle de $B$. varians; ces deux espèces sont également bien représentées sur tous les substrats.

Les supports de -9 et $-13 \mathrm{~m}$ sont colonisés, au début de l'été, par une abondante population de Lyngbya sp. A la même époque, cette espèce est également bien développée sur les tiges de Nitella où elle forme des touffes visibles à l'œeil nu. Elle disparaît pratiquement dès la fin du mois d'août tant sur les substrats naturels qu'artificiels. Exception faite pour cette espèce, les Cyanophycées sont très peu représentées dans les communautés algales des substrats artificiels. Oscillatoria sp. ( -1 et $-4 \mathrm{~m}$ le $13-\mathrm{IX})$ et Clastidium setigerum (presque tous les substrats après 4 mois d'immersion) ne forment jamais de biomasses importantes.

\subsubsection{Comparaison avec les substrats Naturels.}

Les communautés algales qui colonisent les feuilles de polyéthylène ont une composition spécifique très voisine de celle qui a été décrite sur les substrats naturels (Besch et al. 1972; Backhaus en prép.; tableau II); on retrouve pratiquement les mêmes espèces dominantes sur les deux types de substrats. Il apparaît cependant des différences d'ordre qualitatif et quantitatif : 
-- Quelques espèces, abondantes sur les rochers de la zone littorale, sont rares (Cymbella spp. Nitzchia spp.) ou totalement absentes (Nostoc zeterstedtii, Schizothrix spp., Calothrix spp., Pleucapsa spp.) sur les supports artificiels. Ce fait, déjà signalé dans la zone littorale du lac de Port-Bielh (Besch et al. 1972), rejoint les observations de Castenholz (1960) et Neal et al. (1967); il traduit peut-être une sélectivité du polyéthylène vis-à-vis de certaines espèces. Il est vraisemblable que ces différences affectent surtout les formes à croissance lente qui parviennent plus difficilement à coloniser les substrats artificiels. Inversement des espèces relativement rares sur les substrats naturels ont été retrouvées en densités appréciables sur les substrats artificiels. Ainsi, Coleochaete scutata et Chaetosphaeridium pringsheimii ne sont pas signalés dans la liste d'algues établie par Backhaus (en prép.) à partir de prélèvements réalisés fin septembre. Le 10 octobre, ces deux espèces atteignaient respectivement des densités de $1-10$ thalles $/ \mathrm{cm}^{2}$ et 10 - 40 thalles $/ \mathrm{cm}^{2}$ sur les feuilles de polyéthylène immergées à -9 et $-13 \mathrm{~m}$.

- Sur le plan quantitatif, il existe également des différences appréciables. Plus de $50 \%$ du biovolume de Diatomées des substrats artificiels sont le fait de $A$. minutissima. L'étude des communautés naturelles avait révélé que cette algue, numériquement dominante à toutes les profondeurs, représentait moins de $10 \%$ du volume des Diatomées (Besch et al. 1972). Inversement, E. arcus ne constitue que 10 à $40 \%$ du biovolume sur les substrats artificiels immergés en zone profonde contre 55 à $90 \%$ sur les tiges de Nitella. Compte tenu du nombre relativement faible de relevés étudiés, on ne peut affirmer que de telles différences sont imputables au type de substrat utilisé. D'une part, les comptages d'algues sur les substrats naturels incluent inévitablement les frustules de Diatomées mortes et vivantes; l'image qu'ils fournissent représente de ce fait une moyenne des communautés accumulées au cours des périodes qui ont précédé l'analyse. D'autre part, ces différences peuvent résulter de la distribution très hétérogène des algues sur le fond. A l'échelle d'un lac, il peut exister des différences quantitatives appréciables selon les points prospectés (Maciolek et Kennedy 1964). Plusieurs expériences (revues dans Sladeckova 1962) ont montré par ailleurs que la quantité de matériel algal accumulé dépendait, pour une même station, de la position des substrats artificiels. Dans le lac de Port-Bielh, les feuilles de polyéthylène disposées sur des supports verticaux à $50 \mathrm{~cm}$ du fond en 1971 étaient très faiblement colonisées après deux mois d'exposition (350 à 1000 cell./ $\mathrm{cm}^{2}$ à $-1 \mathrm{~m}$ ). En 1972 , nous avons placé des substrats identiques au contact $d u$ fond sur des supports horizontaux 
cylindriques. Après deux mois d'immersion, la densité moyenne des Diatomées atteignait $90.10^{3}$ cell./ $\mathrm{cm}^{2}$ à la même profondeur, soit plus de 100 fois celle de l'année précédente. Les dosages de pigments chlorophylliens ont montré cependant qu'après 4 mois d'exposition la quantité d'algues fixée sur les feuilles de polyéthylène était plus faible que sur les rochers avoisinants : entre -1 et $-6 \mathrm{~m}$, la quantité de chlorophylle a est environ 10 fois plus faible sur les supports artificiels $\left(18,2 \mu \mathrm{g} / \mathrm{dm}^{2}\right.$ en moyenne) que sur les cailloux $\left(180 \mu \mathrm{g} / \mathrm{dm}^{2}\right.$ en moyenne $)$.

\subsubsection{Relations entre biovolume, poids SEc ET teneur en Chlo- ROPHYLLE.}

Ving et un échantillons ont été prélevés le 7-XI par râclage du bioderme sur les supports de plexiglas libérés des feuilles de polyéthylène lors des relevés précédents. Les mesures du biovolume, du poids sec et du poids de cendres, réalisées sur les algues concentrées par centrifugation ont permis d'estimer, au seuil de proba-

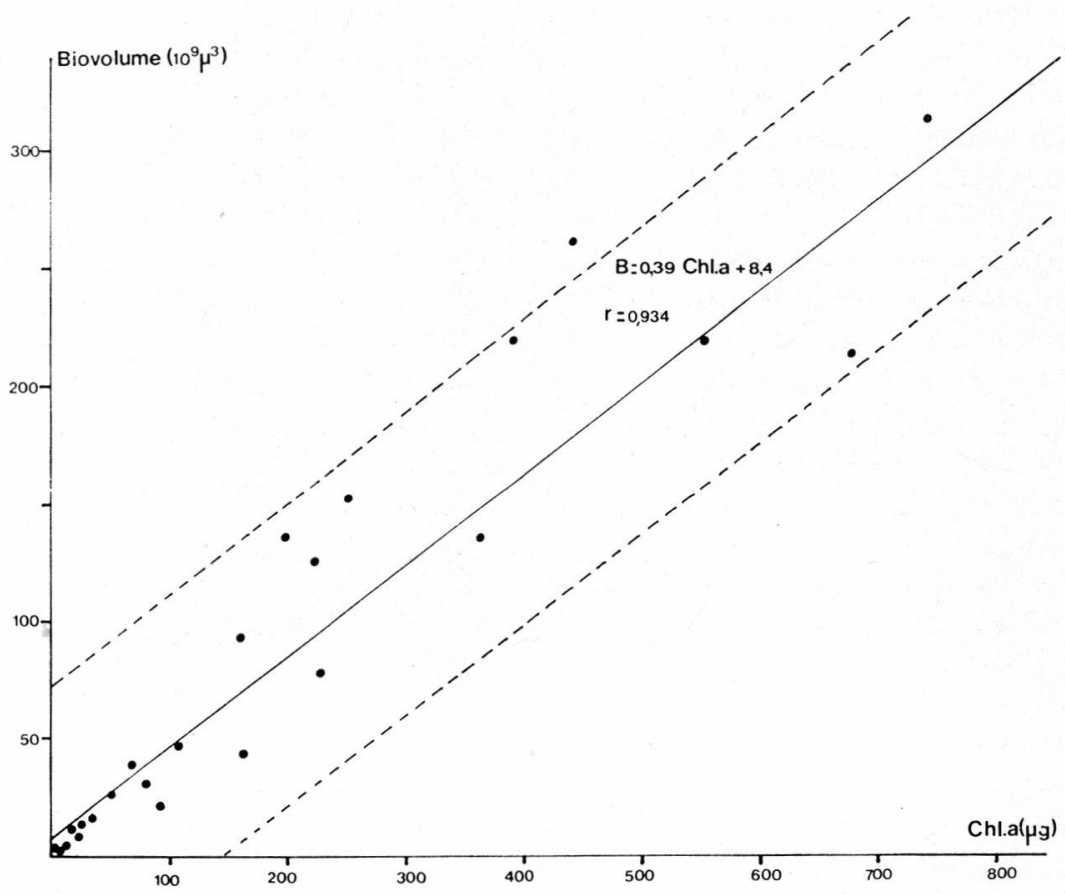

FIG. 5. - Relation entre le biovolume d'algues fixées sur les substrats artificiels et la quantité de chlorophylle a : droite de régression (- - ) et limites de confiance au seuil $5 \%(-\ldots .-)$. 
bilité $\mathrm{P} 0,05$, à $135,4 \pm 15,3 \mathrm{mg}$ le poids sec de $1 \mathrm{ml}$ d'algues et à $\mathbf{4 5} \pm \mathbf{5 , 3} \%$ la quantité de cendres après ignition. Il n'y a aucune différence significative entre les valeurs mesurées sur les substrats immergés en zone littorale et ceux qui ont séjourné en zone profonde. Il existe d'autre part une corrélation hautement significative $\left(r=0,934>r_{0,001}\right)$ entre le biovolume de périphton, estimé à partir des comptages d'algues sur les feuilles de polyéthylène, et la concentration en chlorophylle a des algues recueillies sur ces feuilles (fig. 5). L'utilisation de ces relations permet d'estimer à $2,0 \pm 0,4 \%$ le rapport chlorophylle a/poids sec. Quelques remarques peuvent être faites sur les facteurs de conversion ci-dessous :

a) Si nous adme!tons que la densité des algues vivantes avoisine 1 , le rapport poids sec/poids frais atteint $13,5 \%$, valeur très proche de celle communément admise pour le phytoplancton (10 à $15 \%$ ) et des valeurs moyennes données par Sladecek et Sladeckova (1964) 8 à $9 \%$ ).

b) Certaines algues (Gymbella, Gomphonema) possèdent des pédoncules de fixation gélatineux. Leur volume, non calculé lors des comptages au microscope, constitue une part du biovolume total mesuré par concentration des algues à la centrifugeuse. Il en est de même pour les frustules de Diatomées mortes et pour le matériel non algal (vorticclles, myceliums, particules de vase). La contribution de ces éléments au biovolume total peut modifier légèrement la valeur des relations précédentes. L'examen microscopique des substrats artificiels révèle cependant une quantité de cellules mortes et de particules de vase toujours très faible. La forme adoptée pour les supports et le lavage lors de leur remontée en surface paraît avoir limité leur accumulation. La densité de vorticelles et de mycelium s'avérait également très minime sur tous les substrats examinés.

c) Les Diatomées constituent l'élément essenticl de la biomasse accumulée sur les feuilles de polyéthylène. Leur abondance explique l'augmentation du pourcentage de cendres par rapport à celui qui caraclérise les communautés lithophytiques naturelles (tableau III). La quantité relative de cendres est cependant inférieure à celle donnée par Castenholz (1960) pour du périphyton fixé sur des plaques de verre immergées (65\% en moyenne) mais supérieure à la valeur moyenne de $20 \%$ admise par Sladecek et Sladeckova (1964). On sait que la teneur en carbone par unité de volume est faible chez les Diatomées. Les relations établies par Mullin et al. (1966) et Strathmann (1967) pour les ofrmes planctoniques montrent que la quantité de carbone organique est inversement proportionnelle au volume cellulaire. Appliquées aux Diatomées benthiques, ces relations permettant d'estimer que, pour des 
volumes cellulaires compris entre $120 \mu^{3}$ (A. minutissima) et $6000 \mu^{13}$ (E. arcus.), la teneur en carbone se situe entre 115 et $45 \mathrm{mg} / \mathrm{ml}$ d'algues .

d) La composition des communautés algales étudiées et la méthode utilisée pour mesurer leur biomasse influent sur la valeur du rapport chlorophylle a/poids sec. Les valeurs estimées pour les communautés établies sur les substrats artificiels sont largement supérieures à celles que nous avons obtenues pour les communautés naturelles de la zone littorale (tableau III). Ces différences peuvent être interprétées par la structure du peuplement de ces deux types de substrat. Cependant, en permettant de distinguer les cellules d'algues vivantes du reste du matériel accumulé, les comptages fournissent probablement une estimation de la biomasse plus réaliste que les mesures gravimétriques. Ces dernières ont le plus souvent été utilisées par les différents auteurs qui indiquent des rapports chlorophylle a/poids sec très variables $(0,09$ à $2,4 \%)$ et généralement plus faibles que la valeur donnée ci-dessus.

\subsubsection{Evolution de la Biomasse sur les substrats artificiels.}

La mise en place de substrats artificiels dans le fond d'un lac nous renseigne sur la vitesse moyenne d'accumulation du matériel végétal. Celle-ci résulte de la colonisation des substrats et de la croissance du matériel fixé. Puisque ces deux phénomènes sont indissociables, il n'est pas possible d'estimer le taux de croissance d'une communauté déjà établie. On peut cependant décrire la dynamique de l'accumulation de matériel algal sur une série de substrats dont la durée d'exposition augmente. Les variations de biomasse, dues essentiellement aux espèces dominantes de la microflore benthique, fournissent donc un indice de la potentialité de croissance de ces espèces c'est-à-dire de leur productivité. La comparaison de ces variations à différentes profondeurs donne ainsi une idée de l'importance relative de la productivité du périphyton dans les diverses zones du lac

\subsubsection{Evolution de la biomasse en fonction de la durée d'expo- sition.}

La plupart des auteurs s'accordent à reconnaître que les résultats fournis par les substrats artificiels dépendent, dans une certaine mesure, de la durée d’immersion de ces substrats. Au-delà d'une certaine limite, variable selon les milieux étudiées, on observe généralement une stabilisation ou une diminution de la biomasse fixée consécutive aux pertes de matériel algal par décro- 
chage. L'évolution quantitative du biovolume de périphyton fixé sur les feuilles de polyéthylène immergées entre -1 et $-16 \mathrm{~m}$ le 9-VII-72 et relevées à la fréquence d'une feuille par mois est décrite dans la figure 4. Exception faite des populations de Cyanophycées (essentiellement Lyngbya sp) dont le développement, relativement éphémère, est limité à la zone -9 à $-13 \mathrm{~m}$, les modalités de croissance sont à peu près identiques sur tous les substrats. Nous avons vu par ailleurs (tableau VI) que le périphyton fixé présente pratiquement la même composition spécifique à toutes les profondeurs. Dans un but comparatif, nous avons donc limité cette analyse aux Chlorophycées et aux Diatomées qui composent la quasi totalité de la biomasse sur tous les substrats.

Le biovolume (B) de périphyton présent sur un substrat résulte des gains quotidiens (fixation et développement des algues) diminués des pertes simultanées (respiration, mort et décrochage). Son évolution en fonction du temps ( $t$ ), mesuré depuis la date d'immersion des substrats, est représentée dans la figure 6 en coordonnées

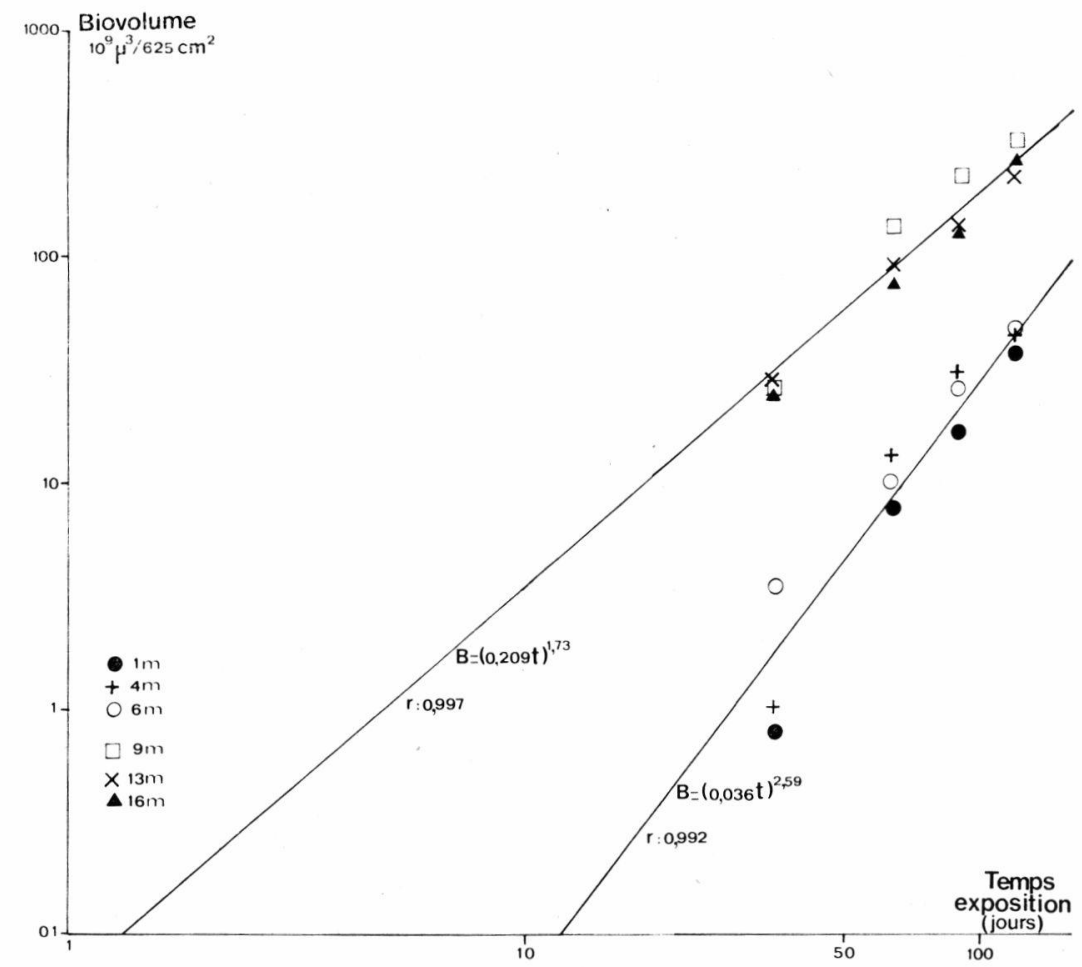

FIG. 6. - Relations entre le biovolume de périphyton fixé (Diatomées + Chlorophycées) et la durée d'exposition des substrats artificiels. 
logarithmiques. Les biovolumes mesurés tous les mois sur les feuilles de polyéthylène exposées dans la zone littorale $(-1$ à $-6 \mathrm{~m})$ et dans la zone littori-profonde $(-9$ à $-13 \mathrm{~m})$ se groupent le long de deux droites $(r \geqslant 0,992)$. Dans chacune de ces zones, la croissance du périphyton (Chlorophycées + Diatomées) peut être décrite par une équation parabolique de la forme :

$$
B=(k t)^{n}
$$

La dérivée première de cette équation exprime l'accroissement par unité de temps, soit la production nette apparente $(\mathrm{P})$ :

$$
P=\frac{d B}{d t}=n \cdot k^{n} \cdot t^{n-1}=n \cdot k \cdot B^{1-\frac{1}{n}}
$$

Les relations entre la biomasse et son taux de croissance peuvent être exprimées par une fonction puissance de la biomasse. Ceci indique que le rapport entre l'accroissement en poids et les pertes reste constant pendant toute la durée de la colonisation ou qu'il varie comme une fonction puissance de $B$ (Winberg 1971).

Le taux de croissance relatif $\mathrm{P} / \mathrm{B}$ est donné par la formule :

$$
\mathrm{P} / \mathrm{B}=\left(\frac{1}{\mathrm{~B}}\right)\left(\frac{\mathrm{dB}}{\mathrm{dt}}\right)=\mathrm{n} \cdot \mathrm{t}^{-1}=\mathrm{n} \cdot \mathrm{k} \cdot \mathrm{B}^{-\frac{1}{\mathrm{n}}}
$$

Le rapport $\mathrm{P} / \mathrm{B}$ est donc inversement proportionnel au temps d'exposition des substrats. Ce résultat concorde avec les observations de Sladecek et Sladeckova (1964) qui ont montré que l'augmentation de la fréquence d'échantillonnage accroît la valeur du taux de renouvellement. Il est encore inversement proportionnel à une puissance de $B$ : cette relation est identique à celle précédemment décrite pour le phytoplancton du lac de Port-Bielh (Capblancq 1972).

Castenholz (1960) estime que la durée optimale d'immersion des substrats artificiels ne doit pas excéder 2 à 4 semaines, les pertes subies par les comunautés algales fixées augmentant avec la durée d'exposition. Par des examens réguliers de feuilles de polyéthylène immergées dans le Danube, Backhaus (1967) arrive à une conclusion iden'ique. Szczepanski et Szczepanska (1966) ont observé qu'après 14 jours de colonisation, la biomasse de périphyton se stabilise pendant 4 semaines. De même la courbe de croissance du périphyton sur des plaques immergées dans un ruisseau artificiel atteignait un plateau après deux mois d'exposition (Mc Intire et Phinney, 1965).

Les modalités de la croissance du périphyton sur les substrats artificiels immergés au lac de Port-Bielh différent assez nettement 
avec ces observations. Les relations précédentes semblent indiquer que les pertes affectent peu les communautés fixées, quelles que soient la durée d'exposition et la biomasse établie. Une augmentation notable des pertes entraînerait en effet une diminution du taux de croissance apparent et l'évolution des biomasses se traduirait par une courbe d'allure sigmoïde. Le risque de décrochage accidentel du bioderme lors du relevé augmentant en même temps que l'épaisseur de périphyton, la densité de formes filamenteuses moins adhérentes et la profondeur d'immersion des substrats, ces courbes devraient donc apparaître plus rapidement pour les substrats de la zone profonde. Ces résultats, joints aux différences quantitatives évoquées précédemment, suggèrent que la durée de la colonisation nécessaire pour atteindre une biomasse optimale sur les substrats artificiels excède 4 mois dans le lac de PortBielh, indice d'une productivité relativement faible.

\subsubsection{2. - Taux de production.}

Il est assez difficile d'appliquer les relations précédentes au calcul de la productivité de la microflore benthique du lac de PortBiclh. En effet :

- contrairement aux substrats artificiels, les substrats naturels ne sont jamais dépourvus d'algues quelle que soit la saison.

- la comparaison, en zone littorale, de la biomasse présente sur les rochers et sur les substrats immergés à leur contact depuis 4 mois a révélé des différences qualitatives et quantitatives appréciables.

- même si les relations établies entre la biomasse et son taux de croissance étaient identiques sur tous les types de substrats, l'évaluation de la quantité d'algues par unité de surface de support naturel (blocs de granite, tiges de Nitella) reste un problème difficile à résoudre.

-- qu'elles soient exprimées en biovolume, en poids sec ou en quantité de pigments, les variations globales de la biomasse de périphyton décrivent imparfaitement les modalités de croissance. Ainsi, la stabilisation ou la diminution de la biomasse totale entre deux relevés successifs peut résulter soit du ralentissement de la croissance de l'ensemble de population, soit du déclin d'une ou de plusieurs populations compensé, totalement ou en partie, par la croissance des autres. Dans ce dernier cas, il est évident que la production n'est pas nulle.

Seuls les comptages d'algues permettent une analyse distincte des courbes de croissance de chaque population. Un exemple de leur succession saisonnière sur deux substrats, l'un de la zone 
littorale $(-4 \mathrm{~m})$, l'autre de la zone littori-profonde $(-13 \mathrm{~m})$, est illustré dans la figure 7. Chaque courbe permet d'estimer le taux de croissance moyen de chaque population de la communauté pendant la période comprise entre deux relevés successifs. La somme de ces taux de croissance spécifiques correspond au taux de production net de la communauté. Toutefois, la forme des courbes de croissance et le niveau de l'asymptote dépendant en partie de la fréquence des relevés, ces résultats n'ont qu'une valeur approximative. Puisque les populations dominantes et leur succession dans le temps sont pratiquement les mêmes à toutes les profondeurs, ils fournissent cependant un bon indice de la productivité relative du périphyton aux diverses profondeurs du lac.

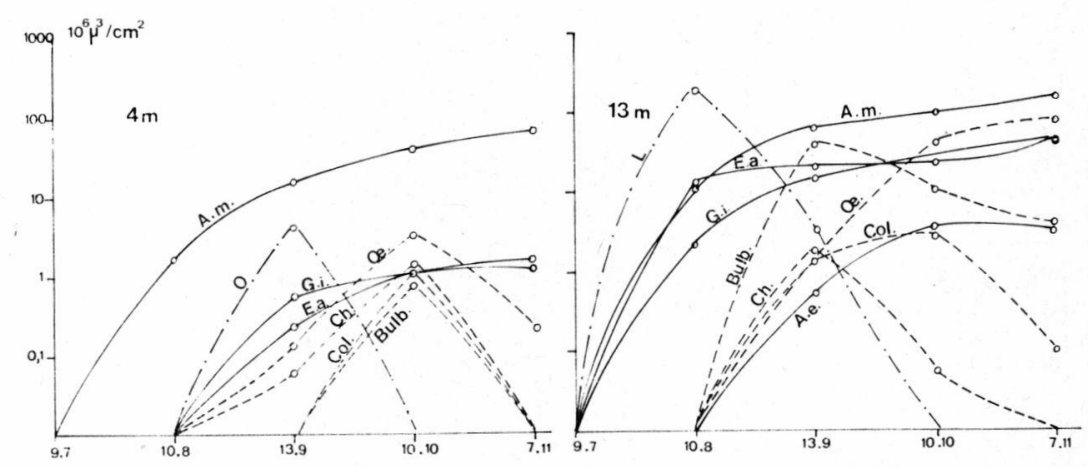

Fig. 7. - Evolution du biovolume des populations dominantes sur les substrats artificiels immergés à -4 et à $-13 \mathrm{~m}$. A. m. : Achnanthes minutissima; E. a : Eunotia arcus; G.i : Gomphonema intricatum; A. e: Anomoeoneis exilis; Bulb. : Bulbochaete varians; Oe : Oedogonium spp.; Col. : Coleochaete scutata; Ch. : Chaetosphaeridium pringsheimii; O : Oscillatoria; L. : Lyngbya sp.

Les résultats des calculs, effectués pour chaque profondeur et pour chaque intervalle de temps entre deux relevés successifs, sont présentés dans le tableau VII. Les taux moyens de production journaliers y sont exprimés en $\mathrm{mg}$ poids $\mathrm{sec} / \mathrm{m}^{2}$ de substrat/jour au moyen de la relation établie précédemment $\left(10^{9} \mu^{3}=0,135 \mathrm{mg}\right.$ de poids sec). Trois observations peuvent être faites à la lecture de ce tableau :

a) L'évolution des taux moyens de production au cours des 4 mois d'été est fonction de la nature des communautés qui colonisent les substrats. A $-\mathbf{1} \mathrm{m}$, les feuilles sont peuplées presque exclusivement par $A$. minutissima (tableau VI). La biomasse fixée augmente progressivement au cours de l'été comme une fonction puissance du temps d'immersion des substrats (fig. 6). Une relation identique décrit la croissance des populations d'A. minutissima, 
G. intricatum et $E$. arcus sur les substrats immergés aux autres profondeurs. La relation de proportionnalité qui existe, en conséquence, entre le taux de croissance de ces trois espèces et une certaine puissance de leur biomasse semble indiquer une absence, chez les Diatomées, de fluctuations du taux de production au cours de l'été. Ce modèle général est modifié par le développement de populations plus éphémères de Lyngbya sp. $(-9$ et $\longrightarrow 13 \mathrm{~m}$ en juillet), $B$. varians et $C$. scutata $(-4$ à $-16 \mathrm{~m}$ en août et septembre) et Oedogonium spp. ( -9 à $-16 \mathrm{~m}$ en octobre).

b) Il existe une différence très nette, déjà mise en évidence dans les figures 4 et 6 , entre les zones littorale $(0 \grave{a}-6 \mathrm{~m})$ et littori-profonde. Les taux de production sont, en moyenne, 10 fois supérieurs dans la zone colonisée par les Nitella, les valeurs maximales correspondant presque toujours à la profondeur de $-9 \mathrm{~m}$. L'analogie avec les profils verticaux de productivité du phytoplancton (Capblancq 1972) est assez frappante. La différence notable qui apparaît ici entre les deux zones nous incite à penser que la nature du fond joue un rôle prépondérant dans la productivité des algues benthiques. Les échanges entre le substrat et l'eau susjacente sont probablement très réduits au contact des rochers granitiques de la zone littorale. Comme pour le phytoplancton de surface, le ralentissement de la productivité peut être interprété par l'action conjuguée des fortes intensités lumineuses et de la faible disponibilité en substances nutritives. Les conditions de développement sont plus favorables à l'interface vase - eau de la zone profonde. D'une part, les échanges qui s'établissent à ce niveau compensent l'appauvrissement de l'eau en éléments nutritifs (phosphates, nitrates); d'autre part, la décomposition des matières organiques de la vase et la respiration des Nitella constituent une source de $\mathrm{CO}_{2}$ utilisable par les algues épiphytes. On sait enfin que la microflore algale est capable, au même titre que les bactéries, d'utiliser les composés organiques solubles produits par le métabolisme des macrophytes hôtes (Allen 1969 et 1971; Wetzel et Allen 1972).

c) En comparaison avec les résultats obtenus par des pesées du périphyton accumulé sur des plaques immergées dans des lacs des Etats-Unis (Newcombe 1950; Castenholz 1960), du Canada (Stockner et Armstrong 1971), de Tchécoslovaquic (Sladecek et Sladeckova 1964) et dans un ruisseau artificiel (Kevern et al. 1966), les taux de production du périphyton du lac de Port-Biclh apparaissent très faibles. La plupart des auteurs cités indiquent des taux moyens de croissance de l'ordre de 100 à $250 \mathrm{mg}$ de poids $\mathrm{sec} / \mathrm{m}^{2} /$ jour soit environ 15 à 40 fois plus que la valeur moyenne du lac de Port-Bielh. 
Tablead VII. - Taux d'accroissement moyen journalier du périphyton sur les substrats artificiels. Les valeurs sont exprimées en $\mathrm{mg}$ Poids $\mathrm{sec} / \mathrm{m}^{2} /$ jour en admettant que $10^{12} \mu^{3}$ d'algues $=135 \mathrm{mg}$ P.S.

\begin{tabular}{|c|c|c|c|c|c|c|c|}
\hline $\begin{array}{c}\text { Période } \\
\text { d'exposition }\end{array}$ & $\begin{array}{c}\text { Durée } \\
\text { d'exposition } \\
\text { entre } 2 \text { relevés } \\
(\mathbf{j})\end{array}$ & $1 \mathrm{~m}$ & $4 \mathrm{~m}$ & $6 \mathrm{~m}$ & $9 \mathrm{~m}$ & $13 \mathrm{~m}$ & $16 \mathrm{~m}$ \\
\hline 9-VII - 10-VIII & 32 & 0,05 & 0,07 & 0,24 & 14,57 & 10,36 & 1,67 \\
\hline 10-VIII - 13-IX & 33 & 0,46 & 0,79 & 0,52 & 6,95 & 4,79 & 3,45 \\
\hline $13-\mathrm{IX}-10-\mathrm{X}$ & 27 & 0,88 & 1,58 & 1,30 & 8,96 & 5,23 & 3,63 \\
\hline $10-\mathrm{X}-7-\mathrm{XI}$ & 28 & 1,70 & 1,43 & 1,12 & 12,61 & 7,00 & 12,41 \\
\hline Moyennes & 30 & 0,77 & 0,97 & 0,79 & 10,77 & 6,84 & 5,29 \\
\hline
\end{tabular}

\subsection{Productivité de Nitella flexilis.}

Il est pratiquement impossible de dissocier les rameaux des Nitella de la microflore algale qui leur est associée lors des mesures des vitesses d'assimilation de ${ }^{14} \mathrm{C}$ en enceintes closes. Les résultats doivent être considérés comme une mesure globale de la productivité de ces deux communautés. Les histogrammes de la figure 8 montrent, pour chacune des 7 séries de mesures réalis ́́es, la valeur du taux de photosynthèse aux différentes profondeurs. Ce taux, exprimé en quantité moyenne de carbone assimilé/heure d'exposition/unité de poids végétal sec, correspond au «coefficient d'activité »du complexe Nitella flexilis + périphyton.

\subsubsection{Variations EN FonCtion de LA PROFONDEUR.}

La comparaison des histogrammes (fig. 8) révèle que le coefficient d'activité photosynthétique de Nitella flexilis varie en profondeur selon un schéma commun aux 7 séries de mesures réalisées au cuurs ue 1 elé 1912 el caracterise par.

- une activité optimale dans la zone supéricure de distribution de cette algue $(-6$ à $-9 \mathrm{~m})$,

- un minimum, de l'ordre de $35 \%$ à $61 \%$ du taux optimal, entre -12 et $-15 \mathrm{~m}$,

- une très légère remontée du taux de photosynthèse entre - 15 et $-17,5 \mathrm{~m}$, profondeur limite de distribution de Nitella dans le lac.

Cette tendance générale est résumée dans le tableau VIII qui donne, pour 4 tranches de la zone littori-profonde, le taux moyen de photosynthìse, la biomasse moyenne de Nitella flexilis et sa teneur moyenne en chlorophylle a. La comparaison des valeurs relatives de ces trois paramètres aux diverses profondeurs montre 

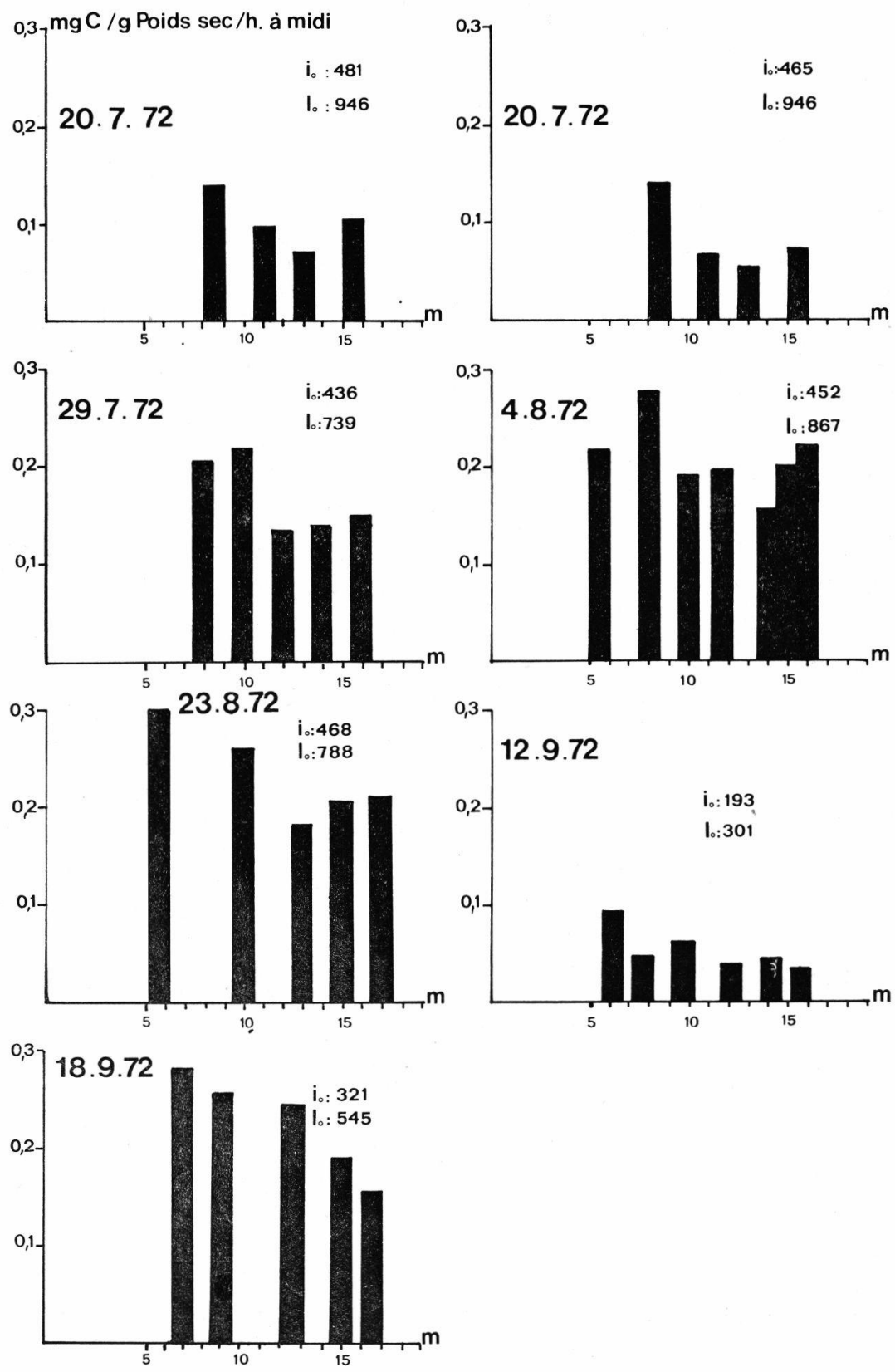

FIG. 8. - Nitella flexilis : activité photosynthétique (mg C/g P.S./h. à midi) à différentes profondeurs et à diverses époques de l'été. L'énergie radiante à la surface du lac pendant l'exposition $\left(i_{0}\right)$ et pendant la journée $\left(I_{0}\right)$ est indiquée sur les figures. 
que le coefficient d'activité photosynthétique varie en raison inverse de la biomasse de Nitella $/ \mathbf{m}^{2}$ de fond et de leur teneur en chlorophylle a. Dans la zone littori-profonde supérieure, Nitella flexilis se présente sous forme de touffes clairsemées à tiges courtes relativement pauvres en chlorophylle. L'assimilation photosynthétique/unité de poids végétal y est environ 1,5 fois plus forte qu'entre -12 et $-15 \mathrm{~m}$, zone de densité optimale de Nitella colonisée par des tiges à la fois plus longues, plus rapprochées et plus riches en chlorophylle.

Tableau VIII. - Nitella flexilis : variations absolues et relatives de l'activité photosynthétique moyenne, de la biomasse et de la teneur en chlorophylle a en fonction de la profondeur.

\begin{tabular}{lrrrr}
\hline Profondeur (m) & $6-9$ & $9-12$ & $12-15$ & $15-17$ \\
\hline Assimilation moyenne $(\mu \mathrm{g} \mathrm{C} / \mathrm{mg}$ P.S./h) & 0,197 & 0,163 & 0,127 & 0,135 \\
\% A. max. & 100 & 82,7 & 64,5 & 68,5 \\
Biomasse moyenne (g P. S./m) & 16,0 & 42,5 & 61,6 & 41,5 \\
\% B. max. & 26,0 & 69,0 & 100 & 67,4 \\
Ch!. a moyenne ( $\mu \mathrm{g} / \mathrm{g}$ P. S.) & 2,42 & 2,44 & 2,91 & 3,28 \\
$\%$ Chl. a max. & 73,8 & 74,4 & 88,7 & 100 \\
\hline
\end{tabular}

Ces observations peuvent être rapprochées de celles recueillies dans l'étude de la croissance du périphyton sur les substrats artificiels. La similitude des courbes de variation de la productivité de Nitella (tableau VIII) avec celles du périphyton (tableau VII) suivant la profondeur suggère que les algues épiphyles jouent probablement un rôle essentiel dans l'activité de la communauté. Les résultats peuvent être interprétés de la façon suivante. On peut penser que la quantité de périphyton/unité de poids de Nitella est d'autant plus faible que la densité et la longueur des tiges du végétal hôte sont élevées. Westlake (1964 et 1966) a montré que l'autoextinction de la lumière est important dans les herbiers et qu'il existe une relation entre la biomasse de végétaux et le coefficient d'extinction vertical spécifique. Au pied des touffes denses, la lumière peut être réduite à moins de $0,1 \%$ de l'intensité de surface et se compose presque exclusivement de radiations vertes. Dans les touffes denses de Nitella en zone profonde, ce phénomène doit entraîner une extinction quasi totale de la lumière, déjà fortement atténuée par la couche d'eau. En conséquence, il est probable que la croissance du périphyton se réduise aux parties apicales des tiges, les seules à bénéficier d'un éclairement suffisant. Inversement, plus on se rapproche de la surface et plus les conditions lumineuses deviennent favorables par suite de la diminution de la 
couche d'eau traversée et de la densité de la végétation sur le fond. L'augmentation de la teneur en chlorophylle a en profondeur (tableau V) est une réponse adaptative de Nitella à la diminution de la lumière et aux changements de sa composition spectrale, analogue à celle observée chez les plantes terrestres qui vivent dans la lumière bleue-verte des sous-bois (Gessner 1955).

\subsubsection{VARIATIONS DANS LE TEMPS.}

A l'exception des mesures réalisées le 12-IX-1972 par temps tris nuageux, les taux moyens de photosynthèse paraissent légèrement plus faibles en juillet $(0,100$ et $0,168 \mu \mathrm{gC} / \mathrm{mg}$ P.S./h) qu'en août et septembre ou ils se maintiennent à un niveau relativement constant $(0,210$ à $0,230 \mu \mathrm{gC} / \mathrm{mg}$ P.S. $/ \mathrm{h})$. Les mesures réalisées sur deux demi-journées le 20-IX-1972 ont montré, par ailleurs, l'existence d'une légère dissymétrie dans la courbe journalière de photosynthèse, analogue à celle précédemment observée pour le phytoplancton; les taux d'assimilation moyens entre le lever du jour et $13 \mathrm{~h}$ sont environ 1,25 fois plus forts que ceux de l'après-midi. Les expériences réalisées dans des flacons parallèles ont montré cependant que les écarts entre deux échantillons de Nitella de même profondeur peuvent égaler, voire dépasser, les variations entre les diverses mesures. Ces écarts peuvent être attribués d'une part aux lifférences d'activité photosynthétiques entre les parties apicales ct basales des tiges de Nitella, d'autre part à l'hétérogénéité de distribution des algues épiphytes sur ces tiges.

L'évolution de l'activité photosyn'hétique correspond cependant assez bien à celle de la croissance du périphyton sur les substrats artificiels, les valeurs d'août et de septembre coïncidant avec la phase de développement des Chlorophycées.

\subsubsection{Relations ENTRE L'ACtivité PHOtosynthétique ET L'Éclai- REMENT}

Les résultats des diverses mesures, exprimés par unité de poids végétal sec $(\mu \mathrm{gC} / \mathrm{mg}$ P.S./h) et par rapport à la quantité de chlorophylle a $(\mu \mathrm{gC} / \mu \mathrm{g} \mathrm{Chl}$. a/h) ont été représentés dans la figure 9 en fonction de l'intensité lumineuse moyenne à la profondeur de mesure pendant la durée d'exposition. Cetle dernière a été calculée à partir des enregistrements fournis par un solarigraphe (Kipp \& Zonen) placé près du lac et les valeurs du coefficient d'extinction (Capblancq 1972).

Il n'existe aucune relation apparente entre l'assimilation par unité de poids végétal sec et l'intensité lumineuse (fig. 9 a). La dispersion des points montre que, pour une énergie lumineuse 

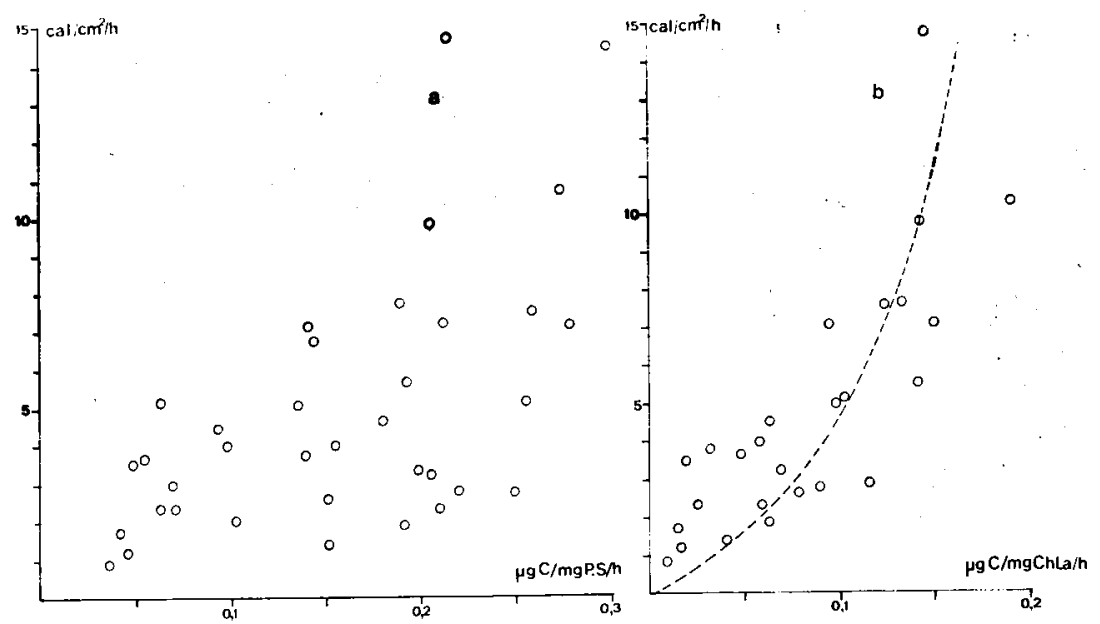

FIG. 9. - Nitella flexilis : relations entre l'énergie lumineuse $\left(\mathrm{I}_{z} \mathrm{cal} / \mathrm{cm}^{2} / \mathrm{h}\right.$, à midi) et l'activité photosynthétique exprimée par rapport au poids sec (a) et par rapport à la teneur en chlorophylle a (b). La courbe en pointillé représente l'équation de la forme $p=p_{\text {max. }} \frac{I_{z}}{I_{z}+K}$ ajustée par la méthode
des moindres carrés.

supérieure à $1,5 \mathrm{cal} / \mathrm{cm}^{2} / \mathrm{h}$, conditions généralement réalisées jusqu'à une profondeur de $18 \mathrm{~m}$, les variations entre les mesures dépendent davantage des échantillons prélevés que de l'énergie lumineuse disponible. La relation entre l'énergie lumineuse et l'assimilation par unité de chlorophylle a est plus nelte. Le nuage de points s'étire approximativement selon une courbe hyperbolique analogue à celle qui décrit la loi de vitesse de réaction enzymatique (équation de Michaelis-Menten). Pour de faibles valeurs de l'intensité lumineuse, le taux d'assimilation augmente proportionnellement à I; il tend vers une valeur optimale (A max) lorsque I augmente. L'enrichissement en chlorophylle de Nitella en profondeur compense ce phénomène si bien que, dans toute la zone colonisée par cette algue, le coefficient d'activité apparaît indépendant de l'énergie lumineuse disponible.

Tableau IX. - Nitella flexilis : assimilation moyenne de carbone à l'obscurité exprimée, aux différentes profondeurs, en valeur absolue, par rapport à l'assimilation optimale et par rapport à l'assimilation photosynthétique.

\begin{tabular}{|c|c|c|c|c|}
\hline Profondeur (m) & $6-9$ & $9-12$ & $12-15$ & $15-17,5$ \\
\hline Assimilation à l'obscurité ( $\mu \mathrm{g} \mathrm{C} / \mathrm{mg}$ P.S./h.) & 0,026 & 0,016 & 0,011 & 0,010 \\
\hline $\begin{array}{l}\% \text { A. } \max \\
\text { A. obsc. }\end{array}$ & 100 & 61,5 & 42,3 & 38,4 \\
\hline A. clair - A. obsc. & 13,2 & 9,8 & 8,6 & 7,4 \\
\hline
\end{tabular}


L'assimilation de carbone dans les flacons obscurs (tableau IX) atteint des valeurs relatives élevées par rapport à celles mesurées pour le phytoplancton (Capblancq 1972). Les variations en fonction de la profondeur sont ici attribuables à l'importance de la microflore fixée sur les tiges de Nitella. On sait que l'assimilation de ${ }^{14} \mathbf{C}$ à l'obscurité reflète en partie l'activité hétérotrophe des communautés bactériennes et algales (Sorokin et Kadota 1972). Plusieurs expériences (Allen 1969 et 1971; Wetzel et Allen 1972) ont montré que les algues sont aussi aptes que les bactéries à utiliser les matières organiques solubles libérées par le métabolisme des plantes hôtes. La diminution de l'assimilation obscure avec la profondeur coïncide bien avec celle de la biomasse de périphyton accumulé sur les substrats entre -9 et $-16 \mathrm{~m}$.

\subsubsection{Importance de LA PROdUCtion PRimaire des algues Benthi- Ques au lac le Port-Bielh.}

La combinaison des résultats des mesures de l'activité photosynthétique (fig. 8) et des estimations de biomasse en zone littoriprofonde (tableau IV) permet d'évaluer la contribution de la flore algale benthique à la production primaire du lac. L'analyse des relations entre le coefficient d'activité photosynthétique et la lumière (fig. 9) montre qu'à la suite d'un phénomène d'adaptation par enrichissement de la teneur en chlorophylle, l'assimilation de carbone/unité de poids sec est pratiquement saturée par la lumière à toutes les profondeurs. Les mesures réalisées sur le phytoplancton établissent que, dans ce cas, l'assimilation journalière est proche de la valeur horaire moyenne pendant la période d'exposition $\times$ longueur du jour $\times 0,9$. Cette relation a été utilisíc pour le calcul de la productivité journalière de la communauté Nitella flexilis + algues épiphytes. Les résultats figurent dans le tableau X.

Tableau X. - Nitella flexilis : productivité journalière moyenne dans la zone littori-profonde du lac de Port-Bielh en été.

\begin{tabular}{|c|c|c|c|c|c|}
\hline Profondeur & $\begin{array}{c}\text { Surface relative } \\
\text { de la zone } \\
\text { littori- } \\
\text { profonde }\end{array}$ & $\begin{array}{c}\text { Biomasse } \\
\left(\mathrm{g} \text { P.S. } / \mathrm{m}^{2}\right)\end{array}$ & $\underset{(\mathrm{mg} \mathrm{C} / \mathrm{g} \text { P.S. } / \mathrm{j})}{\text { P }}$ & $\underset{\left(\mathrm{mg} \mathrm{C} / \mathrm{m}^{2} / \mathbf{j}\right)}{\mathbf{P}}$ & $\begin{array}{l}\text { P } / \mathrm{B} \\
\text { Carbone }= \\
0,4 \times \text { P. S.) }\end{array}$ \\
\hline $6-9$ & $28,25 \%$ & $\begin{array}{c}15,98 \\
(7,7-33,1) \\
42,55\end{array}$ & 2,00 & $\begin{array}{c}31,96 \\
(15,4-66,15) \\
70,20\end{array}$ & 0,005 \\
\hline $9-12$ & $29,30 \%$ & $\begin{array}{c}(29,75-60,85) \\
61,63\end{array}$ & 1,65 & $\begin{array}{c}(49,1-100,4) \\
80,70\end{array}$ & 0,004 \\
\hline $12-15$ & $23,55 \%$ & $\begin{array}{c}(48,53-78,27) \\
41,55\end{array}$ & 1,29 & $\begin{array}{c}(62,6-100,9) \\
56,90\end{array}$ & 0,0032 \\
\hline $15-17$ & $10,35 \%$ & $(27,33-63,15)$ & 1,37 & $(37,4-86,5)$ & 0,0034 \\
\hline $17-19$ & $8,53 \%$ & - & - & 一 & - \\
\hline Moyenne & $11,5 \mathrm{ha}$ & $\begin{array}{c}35,8 \\
(25,1-52,1)\end{array}$ & & $\begin{array}{c}59,94 \\
(41,1-88,5)\end{array}$ & 0.0039 \\
\hline
\end{tabular}


La productivité moyenne des algues benthiques de la zone littori-profonde est de l'ordre de $60 \mathrm{mgC} / \mathrm{m}^{2} /$ jour $\left(41\right.$ à $88 \mathrm{mgC} / \mathrm{m}^{2}$ / jour). Pour l'ensemble du lac, elle représente $15 \%$ à $33 \%$ (23\% en moyenne) de la productivité du phytoplancton. Les données fournies par les substrats artificiels permettent de penser que la contribution des algues lithophytiques de la zone littorale ne modifie pas ces résultats de façon sensible. Nous avons vu (fig. 6 et tableau VII) que leur taux de croissance est, en moyenne, 10 fois moindre qu'en zone littori-profonde : même en admettant que la totalité de la production de cette dernière soit le fait du périphyton, le rapport des laux de croissance des algues sur les feuilles de polyéthylène permet de supposer que la production en zone littorale est inférieure à $10 \mathrm{mgC} / \mathrm{m}^{2} /$ jour. On peut ainsi estimer que les algues benthiques sont responsables de $20 \%$ à $35 \%$ de la production primaire totale du lac de Port-Bielh (30\% en moyenne).

\section{DISCUSSION}

Dans le lac de Port-Bielh, les algues benthiques forment une biomasse végétale importante égale à 140 fois celle du phytoplancton en été. Par opposition aux lacs de basse altitude, la zone littorale est plus pauvre que la zone profonde presque entièrement colonisée par Nitella flexilis. Toutefois, la biomasse est un mauvais indice de la capacité productive des communautés : la contribution des algues benthiques à la production primaire du lac est relativement faible au regard de celle du phytoplancton qui assure $70 \%$ environ de l'assimilation photosynthétique totale. Les mesures du taux de photosynthèse des communautés de la zone profonde et l'étude de la croissance des algues sur des substrats artificiels permettent de conclure que le périphyton joue un rôle important dans la fixation d'énergie par les algues benthiques. L’activité des algues épipéliques n'a pas été évaluée; l'extinction de la lumière par les Nitella limite sans doute leur développement aux zones de vase découverte. Des carottages y ont révélé des populations abondantes de Diatomées (Navicula pupula, Neidium iridis, Pinnularia spp.) et de Desmidiées (Micrasterias rotata).

Dans les lacs anglais du Lake District, Isoetes lacustris et Nitella flexilis caractérisent les comunautés végétales des lacs les moins productifs où leur succession en profondeur est liée à la nature des sédiments (Macan 1970). Leur distribution dans le lac de PortBielh confirme cette observation : Isoetes lacustris crô̂t dans les eaux peu profondes sur un fond de vase et de sable grossier; Nitella flexilis s'établit sur des sédiments plus fins dès la limite de la zone rocheuse, sa biomasse augmentant avec la profondeur 
en même temps que l'épaisseur et la finesse des sédiments. Le coefficient d'activité (taux d'assimilation/unité poids) décroît en raison inverse; il représente en fait l'activité photosynthétique des Nitella et des algues épiphytes. Son évolution selon la profondeur résulte d'un ajustement des communautés végétales aux conditions lumineuses créées en partie par les Nitella elles-mêmes (autoextinction) : dans la zone littori-profonde supéricure le développement du périphyton compense la faible croissance de Nitella; l'importance relative des algues épiphytes décroît en profondeur où, en contrepartie, les Nitella s'enrichissent en chlorophylle. Le taux de renouvellement apparent de Nitella flexilis est de 0,004 en moyenne (tableau $\mathrm{X}$ ); le taux réel, déduction faite de l'activité photosynthétique des algues épiphytes, est sans doute plus faible. Selon Nygaard (1958), dans un lac danois (le Grane Langs $\varnothing$ ), le taux de croissance de cette espèce atteindrait $2 \%$ par jour. Une explication partielle des différences d'activité des deux populations réside dans le fait que cet auteur a mesuré la production d' $\mathrm{O}_{2}$ de la partie apicale des tiges. Wetzel (1964 b) a montré, par ailleurs, que la méthode de l'oxygène donne généralement des taux de production plus forts que la technique $\mathrm{du}{ }^{14} \mathrm{C}$.

L'évaluation de la productivité du périphyton se heurte à de nombreux problèmes méthodologiques. Il s'avère, en particulier, pratiquement impossible d'isoler les algues épiphytes des tiges de Nitella pour mesurer leur activité photosynthétique respective. Une solution adoptée par quelques auteurs (Backhaus 1967; Allen 1971) consiste à mesurer l'activité du périphyton fixé sur des substrats artificiels. Les résultats ne sont pas toujours aisément transposables aux communautés naturelles. Dans le lac de Port-Bielh, le peuplement des supports naturels et artificiels différait de façon parfois notable, tant sur les plans qualitatif que quantitatif. De plus, le rapport entre la quantité d'algues fixées et la durée d'exposition des substrats varie avec les espèces. Par exemple, le taux de croissance des Diatomées augmente pendant toule la période d'exposition comme une fonction puissance de la durée d'immersion. Dans ces conditions, il est difficile de décider quel degré de colonisation représente le stade climacique de la communauté. Dans la mesure où la vitesse de colonisation des surfaces vierges est liée, par une relation qui reste à trouver, à la productivité du périphyton, les substrats artificiels fournissent cependant des valeurs relatives utilisables. En comparaison avec les milieux étudiés à ce jour par une méthode identique, le périphyton du lac de Port-Bielh se caractérise par une faible productivité.

Wetzel (1964 a) a démontré le premier l'importance des comminunautés végétales benthiques dans la production primaire des lacs. Depuis, quelques exemples ont établi que ce phénomène est quasi 
général dans les petits lacs où les régions littorales contribuent souvent pour une part essentielle à la production primaire totale : $43 \%$ dans le Borax Lake en Californie (Wetzel 1964 a), $71,3 \%$ dans le Lawrence Lake au Michigan (Allen 1971), $88 \%$ dans le Marion Lake au Canada (Efford 1972), $23 \%$ dans le lac Mikolajkie en Pologne (Kajak et al. 1972). Par contre, la zone littorale du lac de Port-Bielh se caractérise par une faible productivité : le taux de croissance du périphyton (10 fois moindre qu'en zone profonde) permet de penser que cette zone, avec $36 \%$ de la superficie du lac, contribue pour moins de $5 \%$ à sa productivité totale. Les ressources en éléments nutritifs sont, ici, très faibles : d'une part, les possibilités de régénération de ces éléments à partir du fond rocheux sont pratiquement nulles, d'autre part, les apports allochtones à partir d'un bassin versant constitué d'un sol squelettique sont très réduits. Les échanges au niveau du fond sont vraisemblablement plus intenses au contact des sédiments vaseux de la zone profonde; la transparence élevée des eaux des lacs d'altitude y assure des conditions d'éclairement favorables à l'installa'ion de communautés végétales jusqu'à de grandes profondeurs. Une mousse (Marsupella aquatica) colonise tout le fond d'un lac de montagne en Laponie suédoise, le Latnjajaure $(\mathrm{Zm}=30 \mathrm{~m})$. Bodin et Nauwerck (1968) ont estimé qu'avec la microflore algale benthique et épiphyte, elle assure $40 \%$ de la production primaire annuelle du lac, valeur très proche de celle trouvée pour Nitella flexilis et les algues épiphytes du lac de Port-Bielh.

Ces résultats globaux ne donnent en fait qu'une idée très générale du rôle des communautés végétale benthiques dans les écosystèmes lacustres. Il a été observé par exemple (Hasler et Jones 1949; Vollenweider 1968) qu’il existe un antagonisme entre le développement de la végétation littorale et celui du phytoplancton. Des interactions métaboliques complexes s'établissent entre les macrophytes et la microflore d'algues et de bactéries benthiques et épiphytiques; celles-ci déterminent, du moins en partie, la dynamique des matières organiques dissoutes et celle des éléments minéraux qui lui est intimement liée (Wetzel et Allen 1972). L'activité mélabolique des régions littorales des écosystèmes lacustres apparaît ainsi comme un mécanisme régulateur du fonctionnement de l'écosystème tout entier. Le schéma peut être transposé aux lacs de montagne; nous l'avons résumé dans la figure 10.

Les conditions météorologiques et les caractéristiques pédologiques $d u$ bassin versant réduisent considérablement les apports de substances minérales et organiques. La productivité de la zone pélagique dépend donc essentiellement des possibilités de transfert de substances nutritives des sédiments vers l'eau. Le déficit en oxygène pendant la stagnation hivernale favorise la libération des 


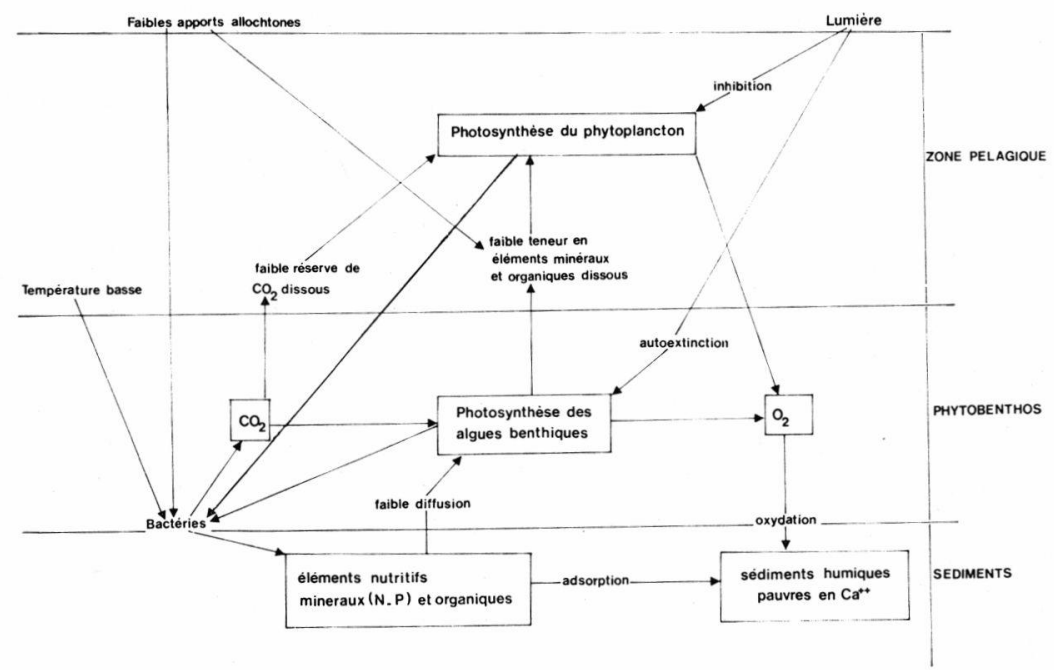

Fig. 10. - Représentation schématique des interrelations entre sédiments, algues benthiques et phytoplancton dans le lac de Port-Beilh.

éléments fixés dans les sédiments. En été, cette zone de contact, entièrement colonisée par la flore algale benthique, est le siège d'une activité métabolique intense. Une première conséquence est l'utilisation des éléments minéraux et organiques, issus des sédiments, par les communautés végétales qui fonctionnent comme un véritable filtre entre le substrat et la zone pélagique. La seconde conséquence résulte de la libération de l'oxygène par la photosynthèse des algues benthiques, qui accélère la formation d'une couche oxydée à la surface des sédiments. On sait (Mortimer 19411942) que les possibilités de migration des sels minéraux de la vase vers l'eau sont d'autant plus réduites que l'épaisseur de la couche oxydée, directement proportionnelle à la concentration en $\mathrm{O}_{2}$ à la surface des sédiments, est forte. Plus les sédiments sont pauvres en bases, plus la capacité d'adsorption de la couche oxydée est élevée. Les sels nutritifs et, en particulier, les phosphates sont alors fortement adsorbés sur les complexes colloïdaux d'hydroxyde ferrique et d'humus. Par ce double mécanisme, la végétation benthique contrôle la diffusion des substances minérales et organiques vers la zone pélagique et, par là même, la productivité du phytoplancton. 


\section{TRAVAUX CITÉS}

AlleEs (H. L.). 1969, - Chemo-organotrophic utilization of dissolved organic compounds by planktic algae and bacteria in a pond. Int. Rev. Ges. Hydrobiol., 54 : 1-33.

Aldeen (H. L..), 1971. - Primary productivity, chemo-organotrophy , and nutritional interactions of epiphytic algae and bacteria on macrophytes in the littoral of a lake. Ecol. Monogr., 41 : 97-127.

Backhaus (D.). 1967. - Ökologische Untersuchungen an den Aufwuchsalgen der obersten Donau und ihrer Quellflüsse. Arch. Hydrobiol. (Suppl.), 30 (4) : 364-399.

Backhaus (D.) (en prép.). - Données écologiques sur les algues benthiques de haute montagne dans les Pyrénées. II. Les algues bleues et vertes.

Baird (J. E.) et WETzel (R. G.) . 1968. - A method for determination of zero thickness activity of 14-C labelled benthic diatoms in sand. Limnol. Oceanogr., $13: 379-382$.

Besch (W. K.), Backhaus (D.), Capblance (J.) el Lavandier (P.). 1972. - Données écologiques sur les algues benthiques de haute montagne dans les Pyrénées. I. Diatomées. Annls limnol., 8 : 103-118.

Bodin (K.) et Nauwerck (A.). 1968. - Produktionsbiologische Studien über die Moosvegetation eines klaren Gebirgsees. Schweiz. $Z$. Hydrol., $30: 318-352$.

Capblance (J.). 1972. - Phytoplancton et productivité primaire de quelques lacs d'altitude dans les Pyrénées. Annls limnol., 8 : 231-321.

Capblance (J.) et Laville (H.). 1968. - Etude morphométrique et physico-chimique de neuf lacs du massif de Néouvielle (Hautes-Pyrénées). Annls limnol., $4: 275-324$.

Capblance (J.) et Lavilide (H.). 1972. - Etude de la productivité du lac de Port-Bielh (Pyrénées Centrales). Proc. IBP-UNESCO Symposium on Productivity Problems of Freshwaters, Kazimierz-Dolny, Poland, May 6-12, 1970 : 73-88.

Cisten holz (R. W.). 1960. - Seasonal changes in the attached algae of freshwater and saline lakes in the lower Grand Coulee. Limnol. Oceanogr., 5 : $1-28$.

Castenhoiz (R.'W.). 1961. - An evaluation of a submerged glass method of estimating production of attached algae. Verh. Internat. Verein. Limnol., $14: 155-159$.

Cooke (W. B.). 1956. - Colonization of artificial bare areas by microorganisms. Bot. rev. $22: 613-618$.

Dumont (H. J.). 1969. - A quantitative method for the study of periphyton. Limnol. Oceanogr., $14: 303-307$.

EFFord (I. E.). 1972. - An interim review of the Marion lake project. Proc. IBP-UNESCO Symposium on Productivity of Freshwaters. Kazimierz-Dolny, Poland, May 6-12, 1970: 89-109.

Gessner (F.). 1955. - Hydrobotanik. Die physiologischen Grundlagen der Pflanzenverbreitung im Wasser. I. Energiehaushalt. Berlin, VEB, Deuscher Verlag der Wissenschaften : $517 \mathrm{pp}$.

Gessner (F.). 1959. - Hydrobotanik. Die physiologischen Grundlagen der Pflanzenverbreitung in Wasser. II. Stoffhaushalt. Berlin, VEB, Deutscher Verlag der Wissenschaften : $701 \mathrm{pp}$. 
GRøNTved (J.). 1960. - On the productivity of microbenthos and phytoplankton in some Danish Fjords. Medd. Dan. Fisk. Havunders. $3: 55-92$.

Hartman (R. T.) et Brown (D. L.). 1967. - Changes in the composition of the internal atmosphere of submerged vascular hydrophytes in relation to photosynthesis. Ecology, $48: 252-258$.

HAsi.er (A.) et Jones (E.). 1949. - Demonstration of the antagonistic. action of large aquatic plants on algae and Rotifers. Ecology, $30: 259-364$.

Hunding ('C.). 1971. - Production of benthic microalgae in the littoral zone of a eutrophic lake. Oikos, $22: 289-397$.

Hunding (C.) et Hargrave (B. T.). 1973. - A comparison of benthic microalgal production measured by $\mathrm{C}_{14}$ and oxygen methods. J. Fish. Res. Board Can. 30:309-312.

Kajak (Z.), Hillbricht Ilikowska (A.) et Pieczzynska (E.). 1972. - The production processes in several Polish lakes. Proc. IBP-UNESCO Symposium on Productivity Problems of Freshwaters, KazimierzDolny, Poland, May 6-12, $1970: 128-147$.

Kevern (N. R.), Wilhm (J. L.) et Van Dyne (G. M.). 1966. - Use of artificial substrata to estimate the productivity of periphyton. Limnol. Oceanogr., 11 : 499-502.

LORENZEN (C. J.). 1967. - Determination of chlorophyll and pheopigments : spectrophotometric equations. Limnol. Oceanogr., 12 : 343-346.

Macan (T. T.). 1970. - Biological studies of the English lakes. Longman, London, $260 \mathrm{p}$.

Maciolek (J. A.) et Kennedy (H. D.). 1964. - Spatial variation in periphyton production in a mountain lake at fall overturn. Verh. Internat. Verein. Limnol., $15: 386-393$.

McIntire (C. D.) et Phinney (H. K.). 1965. - Laboratory studies of periphyton production and community metabolism in lotic environments. Ecol. Monogr., 35 : 237-258.

Mulain (M.M.), Sloan (P. R.) et Eppley (R. W.). 1966. - Relationship between organic carbon content, cell volume, and area in phytoplankton. Limnol. Oceanogr., 11 : 307-311.

Neal. (E. C.), Patten (B. C.) et Depoe (C. E.). 1967. - Periphyton growth on artificial substrates in a radioactively contamined lake. Ecology, 48 918-923.

Newcombe (C. L.). 1950. - A quantitative study of attachment materials in Sodon Lake, Michigan. Ecology, $31: 204-215$.

NygaARd (G.). 1958. - On the productivity of the bottom vegetation in Grane Langs $\emptyset$. Verh. Internat. Verein. Limnol., 13 : 155-155.

Pieczynska (E.) et Sizczepanska (W.). 1966. - Primary production in littoral of several Mazurian lakes. Verh. Internat. Verein. Limnol., $16: 372-379$.

SiAdECkOva (A.). 1962. -- Limnological investigation methods of the periphyton ( Aufwuchs 》) community. Bot. Rev., $28: 286-350$.

Sladecek (V.) et Sladeckova (A.). 1964. - Determination of the periphyton production by means of the glass slide method. Hydrobiologia, 23 : 125-158.

Stockner (J. C. et Armstrong (F. A. J.). 1971. - Periphyton of the Experimental Lakes Area, Northwestern Ontario. J. Fish. Res. Board Can., 28 : 215-229. 
Strathman (R. R.). 1967. - Estimating the organic carbon content of phytoplankon from cell volume or plasma volume. Limnol. Oceanogr., $12: 411-418$.

Strickland (J. D. H.) et Parsons (T. R.). 1968. - A practical handbook of seawater analysis. Bull. Fish. Res. Board Can., 167:371 pp.

Sorokin (Y. I.) et Kadota (H.). 1972. - Techniques for the assesment of microbial production and decomposition in frehwaters. IBP Hanbook $\mathrm{N}^{\circ}$ 23, Blackwell Scientific Publications, Oxford : $112 \mathrm{pp}$.

Szczepanski (A.) et Szczepanska (W.). 1966. - Primary production and its dependance on the quantity of periphyton. Bull. Acad. pol. Sci., Ser. Sci. Biol., $14: 45-50$.

Vollenweider (R. A.) et Saman (A. A.). 1958. - A note on the use of $14 \mathrm{C}$ for measuring Carbon assimilation in periphyton. Manuscrit non publié, $4 \mathrm{pp}$.

Vollen weider (R. A.). 1968. - Les bases scientifiques de l'eutrophisation des lacs et des eaux courantes sous l'aspect particulier du phosphore et de l'azote comme facteurs d'eutrophisation. Rapport O.C.D.E. DAS/CSI/68.27 : 128 p.

Welch (E. B.), Emery (R. M.), Matsuda (R. I.) et Dawson (W. A.). 1972. - The relation of periphytic and planktonic algal growth in an estuary to hydrographic factors. Limnol. Océanogr., 17 : 731-737.

Westrake (D. F.). 1963. - Comparisons of plant productivity. Biol. Rev., $38: 385-425$.

WeStlake (D. F.). 1964. - - Light extinction, standing crop and photosynthesis within beds. Verh. Internat. Verein. Limnol., 15:415425.

Westlake (D. F.). 1965. - Some basic data for investigations of the productivity of aquatic macrophytes. Mem. Ist. Ital. Idrobiol., Suppl., $18: 229-248$.

Westlake (D. F.). 1966. - The light climate for plants in rivers. In : Light as an ecological factor (édité par G. C. Evans, R. Rainbridge et $O$. Rackham). Brit. Ecol. Soc. Symp., $n^{\circ}$ 6, 99-120. Blackwell Scientific Publications.

Wetzes (R. G.) 1964 a. -- A comparative study of the primary productivity of higher aquatic plants, periphyton and phytoplankton in a large, shallow lake. Int. rev. ges. Hydrobiol., 49:1-61.

Wetzer. (R. G.). 1964 b. - Primary productivity of aquatic macrophytes. Verh. Internat. Verein. Limnol., $15: 426-436$.

Wetzel (R. G.). 1965. - Techniques and problems of primary productivity measurements in higher aquatic plants and periphyton. Mem. Ist. Ital. Idrobiol., Suppl., $18: 249-267$.

Wetzel (R. G.) et All.en (H. L.). 1972. - Functions and interactions of dissolved organic matter and the littoral zone in lake metabolism and eutrophication. Proc. IBP-UNESCO Symposium on Productivity Problems of Freshwaters, Kazimierz-Dolny, Poland, May 6-12, $1970: 333-347$.

WinBerg (G. G.). 1971. - Methods for the estimation of production of aquatic animals. Academic Press, London and New York, $175 \mathrm{p}$. 


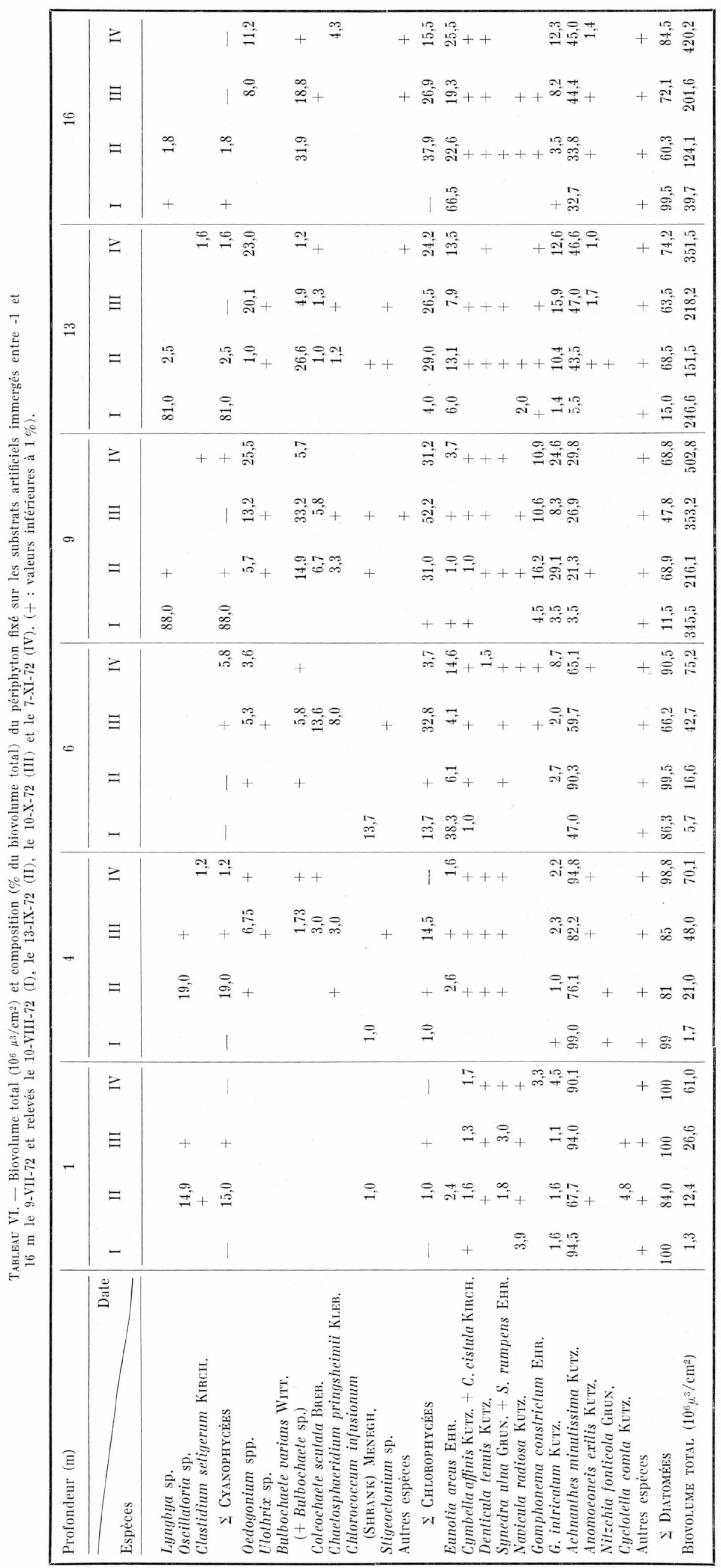

